\title{
A model with ellipsoidal scatterers for polarimetric remote sensing of anisotropic layered media
}

\author{
S. V. Nghiem and R. Kwok \\ Jet Propulsion Laboratory, California Institute of Technology, Pasadena \\ J. A. Kong and R. T. Shin
}

Department of Electrical Engineering and Computer Science and Research Laboratory of Electronics,
Massachusetts Institute of Technology, Cambridge

(Received October 6, 1992; revised June 3, 1993; accepted June 8, 1993.)

\begin{abstract}
This paper presents a model with ellipsoidal scatterers for applications to polarimetric remote sensing of anisotropic layered media at microwave frequencies. The physical configuration includes an isotropic layer covering an anisotropic layer above a homogeneous half space. The isotropic layer consists of randomly oriented spheroids. The anisotropic layer contains ellipsoidal scatterers with a preferential vertical alignment and random azimuthal orientations. Effective permittivities of the scattering media are calculated with the strong flucutation theory extended to account for the nonspherical shapes and the scatterer orientation distributions. On the basis of the analytic wave theory, dyadic Green's functions for layered media are used to derive polarimetric backscattering coefficients under the distorted Born approximation. The ellipsoidal shape of the scatterers gives rise to nonzero cross-polarized returns from the untilted anisotropic medium in the first-order approximation. Effects of rough interfaces are estimated by an incoherent addition method. Theoretical results and experimental data are matched at $9 \mathrm{GHz}$ for thick first-year sea ice with a bare surface and with a snow cover at Point Barrow, Alaska. The model is then used to study the sensitivity of polarimetric backscattering coefficients with respect to correlation lengths representing the geometry of brine inclusions. Polarimetric signatures of bare and snow-covered sea ice are also simulated based on the model to investigate effects of different scattering mechanisms.
\end{abstract}

\section{INTRODUCTION}

Technology in remote sensing has been advanced considerably, especially for airborne and spaceborne radars with multifrequency and multipolarization capabilities. Theoretical models have been developed to interpret multifrequency data for remote sensing of geophysical media. Several approaches including radiative transfer, modified radiative transfer, and analytic wave theories have been considered in model developments [Tsang et al., 1985]. The analytic wave theory, which remains mathematically tractable for some complexity of the media, preserves phase information which is appropriate for the calculation of polarimetric scattering coefficients [Nghiem et al., 1990]. Under the Born approximation for sparse and tenuous media, conventional scattering coefficients have been de-

Copyright 1993 by the American Geophysical Union.

Paper number 93RS01605.

0048-6604/93/93RS-01605\$08.00 rived for isotropic multilayered [Zuniga et al., 1979], anisotropic layered [Lee and Kong, 1985], and isotropic-anisotropic layered [Borgeaud et al., 1986] configurations. Fully polarimetric scattering coefficients have also been calculated for isotropic [Borgeaud et al., 1987] and anisotropic [Borgeaud et al., 1989] layered configurations. In these models, scatterers are spherical or spheroidal and crosspolarized returns come from second- (or higher-) order [Zuniga et al., 1980] or tilted anisotropy [Lee and Kong, 1985]. For denser or less tenuous media the Born approximation needs to be improved. The distorted Born approximation has been applied to random medium modeling [de Wolf, 1971; Rosenbaum and Bowles, 1974; Lang, 1981]. This approximation considers dissipation loss, scattering loss, and the modification of wave speed due to scatterers; thus multiple scattering has been included to some extent. For media with strong permittivity fluctuations, the strong fluctuation theory is used to derive anisotropic effective permittivities [Tsang and Kong, 1981a; Stogryn, 1987]. Tsang and Kong 


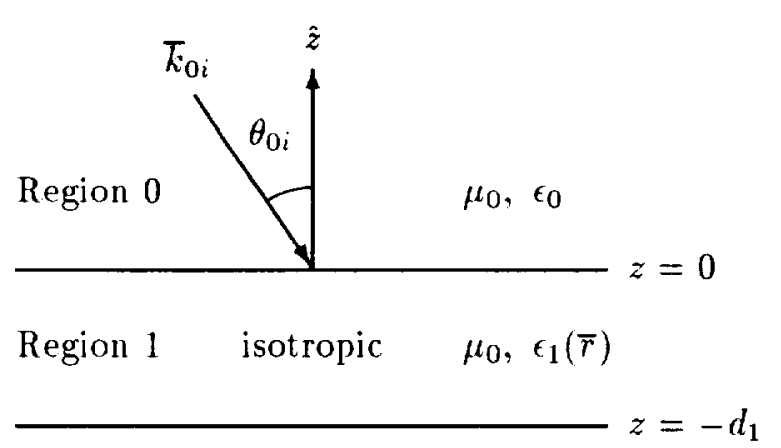

$$
\begin{aligned}
& \text { Region } 2 \text { anisotropic } \mu_{0}, \epsilon_{2}(\bar{r}) \\
& z=-d_{2}
\end{aligned}
$$

Region 3 homogeneous $\mu_{0}, \epsilon_{3}$

Fig. 1. Physical configuration of layered media.

[1981a] further use the derived effective permittivities to calculate copolarized backscattering coefficients under the distorted Born approximation. With this method, conventional backscattering coefficients have been computed for an isotropic halfspace [Tsang et al., 1982], an anisotropic half-space [Tsang and Kong, 1981b], and a configuration for an isotropic layer of spherical scatterers above an anisotropic layer of aligned spheroids [Lin et al., 1987].

Ellipsoidal scatterers have been observed in sea ice in the form of brine inclusions which are oriented preferentially in the vertical direction and randomly in azimuth [Weeks and Ackley, 1982; Gow et al., 1987]. This orientation distribution effectively renders the medium anisotropic. The model in this paper accounts for the ellipsoidal shape, the medium anisotropy in a layered configuration, strong permittivity fluctuations, and fully polarimetric scattering of layered media. The physical configuration is illustrated in Figure 1 where $\theta_{0 i}$ is the incident angle. The covering isotropic layer is composed of randomly oriented spheroids, which have been used to describe scatterers in an inhomogeneous layer [Nghiem et al., 1993]. In the anisotropic layer, scatterers are modeled with an ellipsoidal correlation function with orientations characterized by a probability density function of the Eulerian rotation angles. The strong permittivity fluctuation theory is extended to calculate effective permittivities, and the distorted Born approximation is applied to derive polarimetric scattering coefficients.

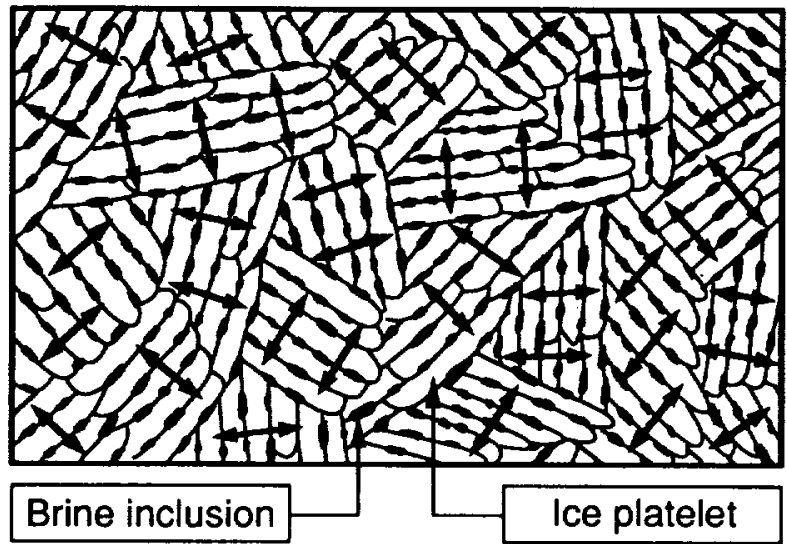

Fig. 2. Illustration of a sea ice horizontal thin section; the arrows represent random horizontal orientations of crystallographic $c$ axes and ellipsoidal brine inclusions.

Theoretical results are compared with measured data at $9 \mathrm{GHz}$ for bare and snow-covered sea ice. Effects of rough interfaces are also estimated by the incoherent addition approach [Lee and Kong, 1985]. Variations of polarimetric backscattering coefficients are studied for various correlation lengths and polarization signatures of sea ice are simulated and discussed for different scattering mechanisms.

\section{EFFECTIVE PERMITTIVITY}

In the isotropic scattering medium of region 1 in Figure 1, embedded scatterers are modeled as spheroids, and the effective permittivity has been derived under the strong fluctuation theory $[\mathrm{Nghiem}$ et al., 1993; Yueh et al., 1990]. In the anisotropic medium (region 2), ellipsoidal scatterers have a preferential alignment resulting in the effective anisotropy. The effective permittivity tensor of the scattering anisotropic medium will be calculated in this section for the ellipsoids with vertical alignment and random azimuthal orientations.

In an inhomogeneous medium such as sea ice, sea water is trapped in an ice medium in the form of brine inclusions which are ellipsoidal as observed in horizontal thin sections and vertical micrographs of sea ice [Weeks and Ackley, 1982; Gow et al., 1987]. The ice tends to grow vertically downward rendering the ellipsoidal inclusions aligned preferentially in the vertical direction. In the absence of sea currents, crystallographic $c$ axes are randomly oriented in azimuth as seen in Figure 2, which depicts 


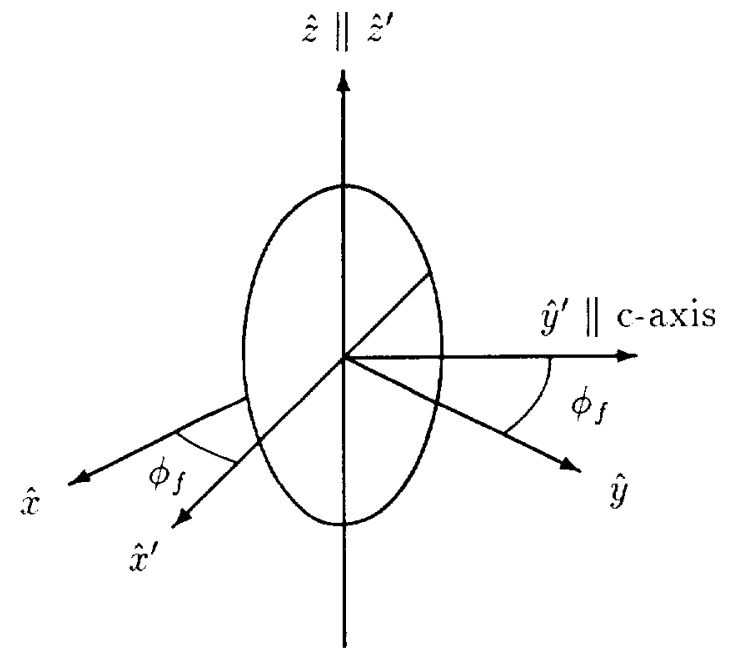

Fig. 3. Geometry of an ellipsoidal scatterer.

$$
\bar{T}=\left[\begin{array}{ccc}
\cos \phi_{f} & \sin \phi_{f} & 0 \\
-\sin \phi_{f} & \cos \phi_{f} & 0 \\
0 & 0 & 1
\end{array}\right]
$$

Effective permittivity tensor $\overline{\bar{\varepsilon}}_{\text {eff }}$ of the inhomogeneous medium is composed of a quasi-static part and a scattering effect part which accounts for the attenuation in wave amplitude and the modification in wave speed due to the inhomogeneities:

$$
\bar{\varepsilon}_{\mathrm{eff}}=\overline{\bar{\varepsilon}}_{g}+\varepsilon_{0}\left[\bar{I}-\bar{\xi}_{\mathrm{eff}} \cdot\langle\bar{S}\rangle\right]^{-1} \cdot \bar{\xi}_{\mathrm{eff}}
$$

where $\overline{\bar{I}}$ is the unit dyad the angular brackets denote ensemble averaging. In (2), auxiliary permittivity $\overline{\bar{\varepsilon}}_{g}$ and dyadic coefficient $\langle\bar{S}\rangle$ are determined by the condition of secular-term elimination [Tsang and Kong, 1981a]. Effective dyadic scatterer $\bar{\xi}_{\text {eff }}$, under the low-frequency approximation, is given by

$$
\begin{aligned}
& {\left[\bar{\xi}_{\mathrm{eff}}\right]_{j m}=\int_{0}^{2 \pi} d \phi_{f} p\left(\phi_{f}\right) \sum_{k, l}^{x, y, z} \Gamma_{\xi j k l m}^{(0)}} \\
& \left.\quad \cdot\left\{k_{0}^{2} \int_{-\infty}^{\infty} d \overline{k^{\prime}}\left[\bar{G}_{g}\left(\bar{k}^{\prime}\right)\right]_{k l} \Phi_{\xi}\left(\overline{k^{\prime}}\right)+[\overline{\bar{S}}]_{k l}\right\}\right|_{\phi_{f}}
\end{aligned}
$$

in which $p\left(\phi_{f}\right)$ is the probability density function of orientation angle $\phi_{f}, \Gamma(0)$ iklm is the variance $[\mathrm{Nghiem}$ et al., 1990], $k_{0}$ is the free-space wave number, and $\Phi_{\xi}$ is the Fourier transform of the normalized local correlation function. The anisotropic Green's function $\overline{\bar{G}}_{g}[$ Tsang and Kong, 1981 $a$ ], which is invariant under the azimuthal Eulerian rotation (1), is expressed in the wave-vector $\bar{k}^{\prime}$ domain as

$$
\begin{aligned}
& \bar{G}_{g}\left(\bar{k}^{\prime}\right)=\frac{1}{\left(k_{x}^{\prime 2}+k_{y}^{\prime 2}\right) D_{o}\left(\bar{k}^{\prime}\right)}\left[\begin{array}{ccc}
k_{y}^{\prime 2} & -k_{x}^{\prime} k_{y}^{\prime} & 0 \\
-k_{x}^{\prime} k_{y}^{\prime} & k_{x}^{\prime 2} & 0 \\
0 & 0 & 0
\end{array}\right] \\
& +\frac{1}{\left(k_{x}^{\prime 2}+k_{y}^{\prime 2}\right) D_{e}\left(\overline{k^{\prime}}\right)}\left[\begin{array}{ccc}
k_{x}^{\prime 2} & k_{x}^{\prime} k_{y}^{\prime} & 0 \\
k_{x}^{\prime} k_{y}^{\prime} & k_{y}^{\prime 2} & 0 \\
0 & 0 & \frac{k_{g \rho}^{2}}{k_{g z}^{2}}\left(k_{x}^{\prime 2}+k_{y}^{\prime 2}\right)
\end{array}\right] \\
& -\frac{\bar{k}^{\prime} \bar{k}^{\prime}}{k_{g z}^{2} D_{e}\left(\overline{k^{\prime}}\right)}
\end{aligned}
$$

where the quantities $D_{o}\left(\bar{k}^{\prime}\right), D_{e}\left(\bar{k}^{\prime}\right), k_{g \rho}^{2}$, and $k_{g z}^{2}$ are given by the following equations for angular frequency $\omega$ and permeability $\mu_{0}$ :

$$
D_{o}\left(\overline{k^{\prime}}\right)=k_{x}^{\prime 2}+k_{y}^{\prime 2}+k_{z}^{\prime 2}-k_{g \rho}^{2}
$$




$$
\begin{aligned}
& D_{e}\left(\overline{k^{\prime}}\right)=k_{z}^{\prime 2}+\frac{k_{g \rho}^{2}}{k_{g z}^{2}}\left(k_{x}^{\prime 2}+k_{y}^{\prime 2}-k_{g z}^{2}\right) \\
& k_{g \rho}^{2}=\omega^{2} \mu_{0} \varepsilon_{g \rho}, \quad k_{g z}^{2}=\omega^{2} \mu_{0} \varepsilon_{g z}
\end{aligned}
$$

As in (2), the effective permittivity tensor has been approximated by truncating the series in the renormalization method [Tsang et al., 1985]. The validity condition for the approximation is $\left|\left[\bar{\xi}_{\mathrm{eff}}(\bar{k})\right]\right|_{j m} \ll 1$, where index $j$ or $m$ can be $x, y$, or $z$.

The ellipsoidal scatterer is described with a normalized local correlation function of the form

$$
R_{\xi}\left(\vec{r}^{\prime}\right)=\exp \left(-\sqrt{\frac{x^{\prime 2}}{l_{x^{\prime}}^{2}}+\frac{y^{\prime 2}}{l_{y^{\prime}}^{2}}+\frac{z^{\prime 2}}{l_{z^{\prime}}^{2}}}\right)
$$

with correlation length $l_{x^{\prime}}, l_{y^{\prime}}$, and $l_{z^{\prime}}$ in the local coordinates corresponding to the minor, the meridian, and the major axes of the scatterer, respectively. In this model, the correlation lengths are related to the effective size and shape of the scatterers. This local correlation function can be reduced to a spheroidal shape for two equal correlation lengths and to a spherical shape for three identical correlation lengths. Applying the Fourier transform in $(5 a)$ yields

$$
\Phi_{\xi}\left(k^{\prime}\right)=\frac{l_{x^{\prime}} l_{y^{\prime}} l_{z^{\prime}}}{\pi^{2}\left(1+k_{x}^{\prime 2} l_{x^{\prime}}^{2}+k_{y}^{\prime 2} l_{y^{\prime}}^{2}+k_{z}^{\prime 2} l_{z^{\prime}}^{2}\right)^{2}}
$$

For random horizontal orientations with no preference in azimuthal direction, the probability density function of orientation is simply

$$
p\left(\phi_{f}\right)=1 /(2 \pi)
$$

To calculate the effective permittivity tensor according to (2), $\overline{\bar{\varepsilon}}_{g}$ and $\langle\bar{S}\rangle$ need to be determined. Because of the global azimuthal symmetry, auxiliary permittivity $\overline{\bar{\varepsilon}}_{g}$ in coordinates $(x, y, z)$ is uniaxial, as indicated previously, and the elements in $\overline{\bar{\varepsilon}}_{g}$ are subject to the condition $\langle\overline{\bar{\xi}}\rangle=0$ such that

$$
\begin{aligned}
\langle\bar{\xi}\rangle & =\int_{0}^{2 \pi} d \phi_{f} p\left(\phi_{f}\right) \bar{T}^{-1} \cdot\left(\left[\begin{array}{ccc}
\xi_{x^{\prime}} & 0 & 0 \\
0 & \xi_{y^{\prime}} & 0 \\
0 & 0 & \xi_{z^{\prime}}
\end{array}\right]\right) \cdot \bar{T} \\
& =\frac{1}{2}\left\langle\left[\begin{array}{ccc}
\xi_{x^{\prime}}+\xi_{y^{\prime}} & 0 & 0 \\
0 & \xi_{x^{\prime}}+\xi_{y^{\prime}} & 0 \\
0 & 0 & 2 \xi_{z^{\prime}}
\end{array}\right]\right)=0
\end{aligned}
$$

Local quantities $\xi_{x^{\prime}}, \xi_{y^{\prime}}$, and $\xi_{z^{\prime}}$ in (7) are related to the elements of dyadic coefficient $\overline{\bar{S}}=\operatorname{diag}\left[S_{x^{\prime}}, S_{y^{\prime}}\right.$, $\left.S_{z^{\prime}}\right]$ by

$$
\begin{aligned}
& \xi_{x^{\prime}}(\varepsilon)=\frac{\varepsilon-\varepsilon_{g \rho}}{\varepsilon_{0}+S_{x^{\prime}}\left(\varepsilon-\varepsilon_{g \rho}\right)} \\
& \xi_{y^{\prime}}(\varepsilon)=\frac{\varepsilon-\varepsilon_{g \rho}}{\varepsilon_{0}+S_{y^{\prime}}\left(\varepsilon-\varepsilon_{g \rho}\right)} \\
& \xi_{z^{\prime}}(\varepsilon)=\frac{\varepsilon-\varepsilon_{g z}}{\varepsilon_{0}+S_{z^{\prime}}\left(\varepsilon-\varepsilon_{g z}\right)}
\end{aligned}
$$

where $\varepsilon$ can take on the value of $\varepsilon_{s}$ in a scatterer or $\varepsilon_{b}$ in the background medium. From (7) and (8), $\varepsilon_{g \rho}$ and $\varepsilon_{g z}$ can be rewritten as

$$
\begin{gathered}
\varepsilon_{g \rho}=\varepsilon_{b}+f_{s} \frac{\varepsilon_{s}-\varepsilon_{g \rho}}{1-f_{s}} \frac{2 \varepsilon_{0}+\left(S_{x^{\prime}}+S_{y^{\prime}}\right)\left(\varepsilon_{s}-\varepsilon_{g \rho}\right)}{2 \varepsilon_{0}+\left(S_{x^{\prime}}+S_{y^{\prime}}\right)\left(\varepsilon_{b}-\varepsilon_{g \rho}\right)} \\
\cdot \frac{\varepsilon_{0}+S_{x^{\prime}}\left(\varepsilon_{b}-\varepsilon_{g \rho}\right)}{\varepsilon_{0}+S_{x^{\prime}}\left(\varepsilon_{s}-\varepsilon_{g \rho}\right)} \frac{\varepsilon_{0}+S_{y^{\prime}}\left(\varepsilon_{b}-\varepsilon_{g \rho}\right)}{\varepsilon_{0}+S_{y^{\prime}}\left(\varepsilon_{s}-\varepsilon_{g \rho}\right)} \\
\varepsilon_{g z}=\varepsilon_{b}+f_{s} \frac{\varepsilon_{s}-\varepsilon_{g z}}{1-f_{s}} \frac{\varepsilon_{0}+S_{z^{\prime}}\left(\varepsilon_{b}-\varepsilon_{g z}\right)}{\varepsilon_{0}+S_{z^{\prime}}\left(\varepsilon_{s}-\varepsilon_{g z}\right)}
\end{gathered}
$$

respectively. The average dyadic coefficient $\langle\overline{\bar{S}}\rangle$ in the global coordinates is obtained by the averaging integration over the probability density function of orientation:

$$
\begin{aligned}
\langle\bar{S}) & =\int_{0}^{2 \pi} d \phi_{f} p\left(\phi_{f}\right) \bar{T}^{-1} \cdot\left[\begin{array}{ccc}
S_{x^{\prime}} & 0 & 0 \\
0 & S_{y^{\prime}} & 0 \\
0 & 0 & S_{z^{\prime}}
\end{array}\right] \cdot \bar{T} \\
& =\frac{1}{2}\left[\begin{array}{ccc}
S_{x^{\prime}}+S_{y^{\prime}} & 0 & 0 \\
0 & S_{x^{\prime}}+S_{y^{\prime}} & 0 \\
0 & 0 & 2 S_{z^{\prime}}
\end{array}\right]=\left[\begin{array}{ccc}
S_{\rho} & 0 & 0 \\
0 & S_{\rho} & 0 \\
0 & 0 & S_{z}
\end{array}\right]
\end{aligned}
$$

Coefficients $S_{x^{\prime}}, S_{y^{\prime}}$, and $S_{z^{\prime}}$ are derived from the secular elimination condition [Tsang and Kong, 1981a] which gives

$$
\begin{aligned}
& S_{x^{\prime}}=\int_{0}^{2 \pi} d \phi \frac{\varepsilon_{0} \gamma_{x}^{2} \cos ^{2} \phi}{2 \pi \varepsilon_{g \rho} a \sqrt{a}}\left[(1+a) \tan ^{-1} \sqrt{a}-\sqrt{a}\right] \\
& S_{y^{\prime}}=\int_{0}^{2 \pi} d \phi \frac{\varepsilon_{0} \gamma_{y}^{2} \sin ^{2} \phi}{2 \pi \varepsilon_{g \rho} a \sqrt{a}}\left[(1+a) \tan ^{-1} \sqrt{a}-\sqrt{a}\right]
\end{aligned}
$$


$S_{z^{\prime}}=\int_{0}^{2 \pi} d \phi \frac{\varepsilon_{0}(1+a)}{2 \pi \varepsilon_{g z} a \sqrt{a}}\left[\sqrt{a}-\tan ^{-1} \sqrt{a}\right]$

In (11) the integrations over $\phi$ can be carried out numerically, and quantities $a, \gamma_{x}$, and $\gamma_{y}$ are defined as

$$
\begin{gathered}
a=\alpha \gamma^{2}-1, \quad \alpha=\frac{\varepsilon_{g z}}{\varepsilon_{g \rho}} \\
\gamma=\frac{1}{l_{z^{\prime}}}\left(\frac{\cos ^{2} \phi}{l_{x^{\prime}}^{2}}+\frac{\sin ^{2} \phi}{l_{y^{\prime}}^{2}}\right)^{-1 / 2} \\
\gamma_{x}=\frac{1}{l_{x^{\prime}}}\left(\frac{\cos ^{2} \phi}{l_{x^{\prime}}^{2}}+\frac{\sin ^{2} \phi}{l_{y^{\prime}}^{2}}\right)^{-1 / 2} \\
\gamma_{y}=\frac{1}{l_{y^{\prime}}}\left(\frac{\cos ^{2} \phi}{l_{x^{\prime}}^{2}}+\frac{\sin ^{2} \phi}{l_{y^{\prime}}^{2}}\right)^{-1 / 2}
\end{gathered}
$$

Also, because of the azimuthal symmetry of the scattering medium, the effective scatterer tensor $\bar{\xi}_{\text {eff }}$ has the uniaxial form

$$
\bar{\xi}_{\text {eff }}=\left[\begin{array}{ccc}
\xi_{\text {eff } \rho} & 0 & 0 \\
0 & \xi_{\text {eff } \rho} & 0 \\
0 & 0 & \xi_{\text {eff }}
\end{array}\right]
$$

which is obtained by substituting the Green's function, the correlation function, and the probability density function of orientation into (3). The integrations are carried out with a procedure involving branch cuts of complex variable functions $[\mathrm{Nghiem}$ et al., 1990]. The following equations determine the diagonal elements of $\overline{\bar{\xi}}_{\text {eff }}$ :

$$
\begin{array}{r}
\xi_{\text {eff } \rho}=\frac{1}{2}\left[\delta_{\xi x^{\prime}}\left(I_{x^{\prime}}+S_{x^{\prime}}\right)+\delta_{\xi y^{\prime}}\left(I_{y^{\prime}}+S_{y^{\prime}}\right)\right] \\
\xi_{\text {eff } z}=\delta_{\xi z^{\prime}}\left(I_{z^{\prime}}+S_{z^{\prime}}\right)
\end{array}
$$

where $I_{x^{\prime}}, I_{y^{\prime}}$, and $I_{z^{\prime}}$ are obtained by comparing (14) to (3). In (14), variance $\delta_{\xi x^{\prime}}, \delta_{\xi y^{\prime}}$, and $\delta_{\xi z^{\prime}}$ are given by

$$
\begin{gathered}
\delta_{\xi x^{\prime}}=\left[\frac{\varepsilon_{b}-\varepsilon_{g \rho}}{\varepsilon_{0}+S_{x^{\prime}}\left(\varepsilon_{b}-\varepsilon_{g \rho}\right)}\right]^{2}\left(1-f_{s}\right) \\
+\left[\frac{\varepsilon_{s}-\varepsilon_{g \rho}}{\varepsilon_{0}+S_{x^{\prime}}\left(\varepsilon_{s}-\varepsilon_{g \rho}\right)}\right]^{2} f_{s}
\end{gathered}
$$

$$
\begin{aligned}
\delta_{\xi y^{\prime}} & =\left[\frac{\varepsilon_{b}-\varepsilon_{g \rho}}{\varepsilon_{0}+S_{y^{\prime}}\left(\varepsilon_{b}-\varepsilon_{g \rho}\right)}\right]^{2}\left(1-f_{s}\right) \\
+ & {\left[\frac{\varepsilon_{s}-\varepsilon_{g \rho}}{\varepsilon_{0}+S_{y^{\prime}}\left(\varepsilon_{s}-\varepsilon_{g \rho}\right)}\right]^{2} f_{s} } \\
\delta_{\xi z^{\prime}} & =\left[\frac{\varepsilon_{b}-\varepsilon_{g z}}{\varepsilon_{0}+S_{z^{\prime}}\left(\varepsilon_{b}-\varepsilon_{g z}\right)}\right]^{2}\left(1-f_{s}\right) \\
+ & {\left[\frac{\varepsilon_{s}-\varepsilon_{g z}}{\varepsilon_{0}+S_{z^{\prime}}\left(\varepsilon_{s}-\varepsilon_{g z}\right)}\right]^{2} f_{s} }
\end{aligned}
$$

respectively. As observed from (14), expressions for $I_{x^{\prime}}, I_{y^{\prime}}$, and $I_{z^{\prime}}$ are necessary to complete the derivation of the anisotropic effective permittivity. The result for $I_{z^{\prime}}$ is

$I_{z^{\prime}}=\int_{0}^{2 \pi} d \phi \frac{-\varepsilon_{0}}{\pi \varepsilon_{g z^{\prime}}}\left(I_{\phi}^{s}+I_{\phi}^{d}\right)$

$I_{\phi}^{s}=-\frac{\alpha \gamma^{2} \sqrt{\alpha \gamma^{2}}}{2 a^{2}}\left[\frac{\sqrt{-\zeta}}{\vartheta_{e}}+\frac{\vartheta_{e}+\zeta}{\vartheta_{e} \sqrt{\vartheta_{e}}}\right.$

$$
\left.\cdot\left(\frac{\pi}{2}-\tan ^{-1} \frac{\sqrt{-\zeta}}{\sqrt{\vartheta_{e}}}\right)\right]
$$

$I_{\phi}^{d}=\frac{\alpha \gamma^{2}}{2 a^{2}}\left[\frac{1+a \nu_{g z^{\prime}}^{2}}{\vartheta_{o}}+\frac{\vartheta_{o}(a+2)-\left(b+a \nu_{g z^{\prime}}^{2}\right)}{\vartheta_{o} \sqrt{\vartheta_{o}}}\right.$

$$
\left.\cdot\left(\frac{\pi}{2}-\tan ^{-1} \frac{1}{\sqrt{\vartheta_{o}}}\right)\right]
$$

$\nu_{g z^{\prime}}^{2}=k_{g \rho}^{2} l_{z^{\prime}}^{2}, \quad k_{g \rho}^{2}=\omega^{2} \mu_{0} \varepsilon_{g \rho}, \quad \zeta=\alpha \gamma^{2} \nu_{g z^{\prime}}^{2}$

$b=\frac{\alpha \gamma^{2}+\zeta}{a}, \quad \vartheta_{o}=b-1, \quad \vartheta_{e}=b+\zeta$

For $I_{x^{\prime}}$ the result is expressed as follows:

$I_{x^{\prime}}=\int_{0}^{2 \pi} d \phi \frac{k_{0}^{2}}{\pi}\left(l_{y^{\prime}}^{2} \gamma_{y}^{4} \sin ^{2} \phi I_{\phi}^{o}+l_{x^{\prime}}^{2} \gamma_{x}^{4} \cos ^{2} \phi I_{\phi}^{e}\right)$

$I_{\phi}^{e}=\alpha\left[\left(I_{1}^{e}-I_{2}^{e^{*}}-I_{3}^{e}\right)+\frac{1}{\zeta}\left(I_{\phi}^{s}+I_{\phi}^{d}-\frac{1}{2}\right)\right]$

$I_{\phi}^{o}=I_{1}^{o}-I_{2}^{o}-I_{3}^{o}$ 


$$
\begin{aligned}
& I_{1}^{e}=\frac{\alpha \gamma^{2} \sqrt{\alpha \gamma^{2}}}{2 \alpha^{2} \vartheta_{e}}\left[-\frac{\sqrt{-\zeta}}{b}+\frac{1}{\sqrt{\vartheta_{e}}}\left(\frac{\pi}{2}-\tan ^{-1} \frac{\sqrt{-\zeta}}{\sqrt{\vartheta_{e}}}\right)\right] \\
& I_{2}^{e}=\frac{1}{2 a \vartheta_{o}}\left[1-\frac{1}{\sqrt{\vartheta_{o}}}\left(\frac{\pi}{2}-\tan ^{-1} \frac{1}{\sqrt{\vartheta_{o}}}\right)\right] \\
& I_{3}^{e}=\frac{\alpha \gamma^{2}}{2 a^{2} \vartheta_{o}}\left[-\frac{1}{b}+\frac{1}{\sqrt{\vartheta_{o}}}\left(\frac{\pi}{2}-\tan ^{-1} \frac{1}{\sqrt{\vartheta_{o}}}\right)\right] \\
& I_{1}^{o}=\left.I_{1}^{e}\right|_{\alpha=1}, \quad I_{2}^{o}=\left.I_{2}^{e}\right|_{\alpha=1}, \quad I_{3}^{o}=\left.I_{3}^{e}\right|_{\alpha=1}
\end{aligned}
$$

The result for $I_{y^{\prime}}$ can also be written in terms of $I_{\phi}^{o}$ and $I_{\phi}^{e}$ as

$I_{y^{\prime}}=\int_{0}^{2 \pi} d \phi \frac{k_{0}^{2}}{\pi}\left(l_{x^{\prime}}^{2} \gamma_{x}^{4} \cos ^{2} \phi I_{\phi}^{o}+l_{y^{2}}^{2} \gamma_{y}^{4} \sin ^{2} \phi I_{\phi}^{e}\right)$

where the relation $I_{y^{\prime}}\left(l_{x^{\prime}}, l_{y^{\prime}}, l_{z^{\prime}}\right)=I_{x^{\prime}}\left(l_{y^{\prime}}, l_{x^{\prime}}, l_{z^{\prime}}\right)$ has been used. Values of square-root and inversetangent functions in the above equations have been chosen in the respective principal Riemann sheets [Nghiem et al., 1990] with branch cuts on the negative real axis of the complex plane.

Substituting $\overline{\bar{\varepsilon}}_{g},\langle\overline{\bar{S}}\rangle$, and $\bar{\xi}_{\text {eff }}$ in (2) yields the uniaxial effective permittivity tensor $\overline{\bar{\varepsilon}}_{\text {eff }}=$ diag $\left[\varepsilon_{\mathrm{eff} \rho}, \varepsilon_{\mathrm{eff} \rho}, \varepsilon_{\mathrm{eff} z}\right]$ whose elements are

$$
\begin{gathered}
\varepsilon_{\text {eff } \rho}=\varepsilon_{g \rho}+\varepsilon_{0} \xi_{\text {eff } \rho} /\left(1-S_{\rho} \xi_{\text {eff } \rho}\right) \\
\varepsilon_{\text {eff } z}=\varepsilon_{g z}+\varepsilon_{0} \xi_{\text {eff } z} /\left(1-S_{z} \xi_{\text {eff } z}\right)
\end{gathered}
$$

As seen from the above expressions, effective permittivity tensor $\bar{\varepsilon}_{\text {eff }}$ is anisotropic with the optic axis in the vertical direction. In the next section the anisotropic effective permittivity is used in the derivation of polarimetric backscattering coefficients with dyadic Green's functions (DGF) for the layered configuration.

\section{SCATTERING COEFFICIENTS}

Consider the configuration in Figure 1 for the following definitions of physical parameters in the layers. Region 0 is the upper half-space with permittivity $\varepsilon_{0}$. Region 1 of thickness $d_{1}$ is an isotropic medium consisted of randomly oriented spheroidal scatterers of permittivity $\varepsilon_{s 1}$ in a background medium of permittivity $\varepsilon_{b 1}$. Region 2 of thickness $d_{2}$ is an anisotropic medium composed of ellipsoidal scatterers of permittivity $\varepsilon_{s 2}$ embedded in a host medium of permittivity $\varepsilon_{b 2}$. Fractional volume of scatterers is $f_{s n}$ for $n=1,2$ in region 1 and 2 , respectively. Region 3 is the underlying homogeneous half-space of permittivity $\varepsilon_{3}$. In this section the distorted Born approximation with permittivities obtained in the last section is applied to derive the complete set of polarimetric backscattering coefficients constituting the covariance matrix or the Mueller matrix, which characterizes polarimetric scattering properties of the layered media.

Polarimetric backscattering coefficients have been defined with ensemble averages of scattered fields [Nghiem et al., 1990]. The averages are calculated with spatial integrations over products of elements of DGF $\overline{\bar{G}}_{0 n}$, field vector $\bar{F}_{n}$, and fourthrank correlation tensor $\overline{\bar{C}}_{\xi n}$ (for $n=1,2$ ) as follows:

$$
\begin{aligned}
& \left\langle\bar{E}_{0 s}(\vec{r}) \bar{E}_{0 s}^{*}(\vec{r})\right\rangle=\sum_{i, j, k, l, m}^{x, y, z} k_{0}^{4} \int_{0}^{\pi} d \psi_{f} \int_{0}^{2 \pi} d \phi_{f} p_{1}\left(\psi_{f}, \phi_{f}\right) \\
& \cdot \int_{V_{1}} d \bar{r}_{1} \int_{V_{1}} d \vec{r}_{1}^{o} C_{\xi i j k l m}\left(\bar{r}_{1}, \vec{r}_{1}^{o} ; \psi_{f}, \phi_{f}\right) \\
& \cdot\left[\left\langle G_{01 i j}\left(\bar{r}, \bar{r}_{1}\right)\right\rangle\left\langle F_{1 k}\left(\bar{r}_{1}\right)\right\rangle\right] \cdot\left[\left\langle G_{01 i l}\left(\bar{r}, \bar{r}_{1}^{o}\right)\right\rangle\left\langle F_{1 m}\left(\bar{r}_{1}^{o}\right)\right\rangle\right]^{*} \\
& +\sum_{i, j, k, l, m}^{x, y, z} k_{0}^{4} \int_{0}^{2 \pi} d \phi_{f} p_{2}\left(\phi_{f}\right) \int_{V_{2}} d \bar{r}_{2} \\
& \cdot \int_{V_{2}} d \vec{r}_{2}^{o} C_{\xi 2 j k i m}\left(\bar{r}_{2}, \bar{r}_{2}^{o} ; \phi_{f}\right) \\
& \cdot\left[\left\langle G_{02 i j}\left(\bar{r}_{,} \bar{r}_{2}\right)\right\rangle\left\langle F_{2 k}\left(\bar{r}_{2}\right)\right\rangle\right] \cdot\left[\left\langle G_{02 i l}\left(\bar{r}, \vec{r}_{2}^{o}\right)\right\rangle\left\langle F_{2 m}\left(\bar{r}_{2}^{o}\right)\right\rangle\right]^{*}
\end{aligned}
$$

where $V_{1}$ is the volume occupied by region 1 and $V_{2}$ is the volume occupied by region 2 . The DGF and the mean fields have been obtained [Nghiem et al., 1990]; the correlation functions need to be specified next. The integrations are then carried out to derive the scattering coefficients.

For region 1 , elements of correlation tensor $\overline{\bar{C}}_{\xi 1}$ in (20) have been defined for the spheroids [Nghiem et al., 1993]. In region 2, elements of correlation tensor $\overline{\bar{C}}_{\xi 2}$ in the global spatial domain are

$C_{\xi 2 j k l m}\left(\bar{r}_{2}, \tilde{r}_{2}^{o}, \phi_{f}\right)=\left\langle\xi_{2 j k}\left(\bar{r}_{2}\right) \xi_{2 l m}^{*}\left(\bar{r}_{2}^{o}\right) \mid \phi_{f}\left(\bar{r}_{2}\right)\right\rangle$

To facilitate the integration of $(20)$, Fourier transforms of correlation functions are introduced for the 
statistically homogeneous scattering medium under consideration:

$$
\begin{gathered}
C_{\xi 2 j k l m}\left(\bar{r}_{2}, \bar{r}_{2}^{o}, \phi_{f}\right)=\int_{-\infty}^{\infty} d \bar{\beta} \Phi_{2 j k l m}(\bar{\beta}) \\
\cdot \exp \left[-i \bar{\beta} \cdot\left(\bar{r}_{2}-\bar{r}_{2}^{O}\right)\right]
\end{gathered}
$$

which is expressed in the global coordinate system $(\hat{x}, \hat{y}, \hat{z})$ and related by Eulerian rotation transformation $\overline{\bar{T}}$, which is determined by (1), to the following nonzero correlations in the local coordinate system $\left(\hat{x}^{\prime}, \hat{y}^{\prime}, \hat{z}^{\prime}\right)$ :

$$
\begin{aligned}
& \Phi_{2 x^{\prime} x^{\prime}}\left(\bar{\beta}^{\prime}\right)=\Phi_{2 x^{\prime} x^{\prime} x^{\prime} x^{\prime}}\left(\bar{\beta}^{\prime}\right)=\delta_{2 x^{\prime} x^{\prime}} \Phi_{\xi_{2}}\left(\bar{\beta}^{\prime}\right) \\
& \Phi_{2 x^{\prime} y^{\prime}}\left(\bar{\beta}^{\prime}\right)=\Phi_{2 x^{\prime} x^{\prime} y^{\prime} y^{\prime}}\left(\bar{\beta}^{\prime}\right)=\delta_{2 x^{\prime} y^{\prime}} \Phi_{\xi_{2}}\left(\bar{\beta}^{\prime}\right) \\
& \Phi_{2 x^{\prime} z^{\prime}}\left(\bar{\beta}^{\prime}\right)=\Phi_{2 x^{\prime} x^{\prime} z^{\prime} z^{\prime}}\left(\bar{\beta}^{\prime}\right)=\delta_{2 x^{\prime} z^{\prime}} \Phi_{\xi_{2}}\left(\bar{\beta}^{\prime}\right) \\
& \Phi_{2 y^{\prime} x^{\prime}}\left(\bar{\beta}^{\prime}\right)=\Phi_{2 y^{\prime} y^{\prime} x^{\prime} x^{\prime}}\left(\bar{\beta}^{\prime}\right)=\delta_{2 y^{\prime} x^{\prime}} \Phi_{\xi_{2}}\left(\bar{\beta}^{\prime}\right) \\
& \Phi_{2 y^{\prime} y^{\prime}}\left(\bar{\beta}^{\prime}\right)=\Phi_{2 y^{\prime} y^{\prime} y^{\prime} y^{\prime}\left(\bar{\beta}^{\prime}\right)}=\delta_{2 y^{\prime} y^{\prime}} \Phi_{\xi_{2}}\left(\bar{\beta}^{\prime}\right) \\
& \Phi_{2 y^{\prime} z^{\prime}}\left(\bar{\beta}^{\prime}\right)=\Phi_{2 y^{\prime} y^{\prime} z^{\prime} z^{\prime}}\left(\bar{\beta}^{\prime}\right)=\delta_{2 y^{\prime} z^{\prime}} \Phi_{\xi_{2}}\left(\bar{\beta}^{\prime}\right) \\
& \Phi_{2 z^{\prime} x^{\prime}}\left(\bar{\beta}^{\prime}\right)=\Phi_{2 z^{\prime} z^{\prime} x^{\prime} x^{\prime}}\left(\bar{\beta}^{\prime}\right)=\delta_{2 z^{\prime} x^{\prime}} \Phi_{\xi_{2}}\left(\bar{\beta}^{\prime}\right) \\
& \Phi_{2 z^{\prime} y^{\prime}}\left(\bar{\beta}^{\prime}\right)=\Phi_{2 z^{\prime} z^{\prime} y^{\prime} y^{\prime}}\left(\bar{\beta}^{\prime}\right)=\delta_{2 z^{\prime} y^{\prime}} \Phi_{\xi_{2}}\left(\bar{\beta}^{\prime}\right) \\
& \Phi_{z^{\prime} z^{\prime} z^{\prime}}\left(\overline{\beta^{\prime}}\right)=\Phi_{2 z^{\prime} z^{\prime} z^{\prime} z^{\prime}}\left(\bar{\beta}^{\prime}\right)=\delta_{2 z^{\prime} z^{\prime}} \Phi_{\xi_{2}}\left(\overline{\beta^{\prime}}\right)
\end{aligned}
$$

where $\Phi_{\xi_{2}}\left(\beta^{\prime}\right)$ is given in $(5 b)$, which is the Fourier transform of $(5 a)$, and variance values of $\delta_{2}$ in (23) are

$$
\begin{gathered}
\delta_{2 x^{\prime} x^{\prime}}=\left|\frac{\varepsilon_{b 2}-\varepsilon_{g 2 \rho}}{\varepsilon_{0}+S_{2 x^{\prime}}\left(\varepsilon_{b 2}-\varepsilon_{g 2 \rho}\right)}\right|^{2}\left(1-f_{s 2}\right) \\
+\left|\frac{\varepsilon_{s 2}-\varepsilon_{g 2 \rho}}{\varepsilon_{0}+S_{2 x^{\prime}}\left(\varepsilon_{s 2}-\varepsilon_{g 2 \rho}\right)}\right|^{2} f_{s 2} \\
\delta_{2 y^{\prime} y^{\prime}}=\left|\frac{\varepsilon_{b 2}-\varepsilon_{g 2 \rho}}{\varepsilon_{0}+S_{2 y^{\prime}}\left(\varepsilon_{b 2}-\varepsilon_{g 2 \rho}\right)}\right|^{2}\left(1-f_{s 2}\right) \\
+\left|\frac{\varepsilon_{s 2}-\varepsilon_{g 2 \rho}}{\varepsilon_{0}+S_{2 y^{\prime}}\left(\varepsilon_{s 2}-\varepsilon_{g 2 \rho}\right)}\right|^{2} f_{s 2} \\
\delta_{2 z^{\prime} z^{\prime}}=\left|\frac{\varepsilon_{b 2}-\varepsilon_{g 2 z}}{\varepsilon_{0}+S_{2 z^{\prime}}\left(\varepsilon_{b 2}-\varepsilon_{g 2 z}\right)}\right|^{2}\left(1-f_{s 2}\right) \\
+\left|\frac{\varepsilon_{s 2}-\varepsilon_{g 2 z}}{\varepsilon_{0}+S_{2 z^{\prime}}\left(\varepsilon_{s 2}-\varepsilon_{g 2 z}\right)}\right|^{2} f_{s 2}
\end{gathered}
$$

$$
\begin{aligned}
& \delta_{2 x^{\prime} y^{\prime}}=\left[\frac{\varepsilon_{b 2}-\varepsilon_{g 2 \rho}}{\varepsilon_{0}+S_{2 x^{\prime}}\left(\varepsilon_{b 2}-\varepsilon_{g 2 \rho}\right)}\right] \\
& \cdot\left[\frac{\varepsilon_{b 2}-\varepsilon_{g 2 \rho}}{\varepsilon_{0}+S_{2 y^{\prime}}\left(\varepsilon_{b 2}-\varepsilon_{g 2 \rho}\right)}\right]^{*}\left(1-f_{s 2}\right) \\
& +\left[\frac{\varepsilon_{s 2}-\varepsilon_{g 2 \rho}}{\varepsilon_{0}+S_{2 x^{\prime}}\left(\varepsilon_{s 2}-\varepsilon_{g 2 \rho}\right)}\right] \\
& \cdot\left[\frac{\varepsilon_{s 2}-\varepsilon_{g 2 \rho}}{\varepsilon_{0}+S_{2 y^{\prime}}\left(\varepsilon_{s 2}-\varepsilon_{g 2 \rho}\right)}\right]^{*} f_{s 2}=\delta_{2 y^{\prime} x^{\prime}}^{*} \\
& \delta_{2 x^{\prime} z^{\prime}}=\left[\frac{\varepsilon_{b 2}-\varepsilon_{g 2 \rho}}{\varepsilon_{0}+S_{2 x^{\prime}}\left(\varepsilon_{b 2}-\varepsilon_{g 2 \rho}\right)}\right] \\
& \cdot\left[\frac{\varepsilon_{b 2}-\varepsilon_{g 2 z}}{\varepsilon_{0}+S_{2 z^{\prime}}\left(\varepsilon_{b 2}-\varepsilon_{g 2 z}\right)}\right]^{*}\left(1-f_{s 2}\right) \\
& +\left[\frac{\varepsilon_{s 2}-\varepsilon_{g 2 \rho}}{\varepsilon_{0}+S_{2 x^{\prime}}\left(\varepsilon_{s 2}-\varepsilon_{g 2 \rho}\right)}\right] \\
& \cdot\left[\frac{\varepsilon_{s 2}-\varepsilon_{g 2 z}}{\varepsilon_{0}+S_{2 z^{\prime}}\left(\varepsilon_{s 2}-\varepsilon_{g 2 z}\right)}\right]^{*} f_{s 2}=\delta_{2 z^{\prime} x^{\prime}}^{*} \\
& \delta_{2 y^{\prime} z^{\prime}}=\left[\frac{\varepsilon_{b 2}-\varepsilon_{g 2 \rho}}{\varepsilon_{0}+S_{2 y^{\prime}}\left(\varepsilon_{b 2}-\varepsilon_{g 2 \rho}\right)}\right] \\
& \cdot\left[\frac{\varepsilon_{b 2}-\varepsilon_{g 2 z}}{\varepsilon_{0}+S_{2 z^{\prime}}\left(\varepsilon_{b 2}-\varepsilon_{g 2 z}\right)}\right]^{*}\left(1-f_{s 2}\right) \\
& +\left[\frac{\varepsilon_{s 2}-\varepsilon_{g 2 \rho}}{\varepsilon_{0}+S_{2 y^{\prime}}\left(\varepsilon_{s 2}-\varepsilon_{g 2 \rho}\right)}\right] \\
& \cdot\left[\frac{\varepsilon_{s 2}-\varepsilon_{g 2 z}}{\varepsilon_{0}+S_{2 z^{\prime}}\left(\varepsilon_{s 2}-\varepsilon_{g 2 z}\right)}\right]^{*} f_{s 2}=\delta_{2 z^{\prime} y^{\prime}}^{*}
\end{aligned}
$$

On the basis of the invariant property of the Fourier transform under the rotation transformation, spectral density $\Phi_{2 j k l m}(\bar{\beta})$ in the global coordinates can be related to those given in (24) by

$$
\begin{gathered}
\Phi_{\xi 2}(\bar{\beta})=\Phi_{\xi_{2}}\left(\beta_{x}^{\prime}=\beta_{x} \cos \phi_{f}+\beta_{y} \sin \phi_{f},\right. \\
\left.\beta_{y}^{\prime}=-\beta_{x} \sin \phi_{f}+\beta_{y} \cos \phi_{f}, \beta_{z}^{\prime}=\beta_{z}\right)
\end{gathered}
$$

In the global coordinates, the rotation transformation together with the above invariant property cast the anisotropic spectral densities into the form 


$$
\Phi_{2 j k l m}(\bar{\beta})=\delta_{2 j k l m} \Phi_{\xi 2}(\bar{\beta})
$$

where variance $\delta_{2 j k l m}$ is dependent on the Eulerian angle $\phi_{f}$ as

$$
\begin{gathered}
\delta_{2 j k l m}=\left(\delta_{2 x^{\prime} x^{\prime}} T_{x x j k}+\delta_{2 y^{\prime} x^{\prime}} T_{y y j k}+\delta_{2 z^{\prime} x^{\prime}} T_{z z j k}\right) T_{x x l m} \\
+\left(\delta_{2 x^{\prime} y^{\prime}} T_{x x j k}+\delta_{2 y^{\prime} y^{\prime}} T_{y y j k}+\delta_{2 z^{\prime} y^{\prime}} T_{z z j k}\right) T_{y y l m} \\
+\left(\delta_{2 x^{\prime} z^{\prime}} T_{x x j k}+\delta_{2 y^{\prime} z^{\prime}} T_{y y j k}+\delta_{2 z^{\prime} z^{\prime}} T_{z z j k}\right) T_{z z l m}
\end{gathered}
$$

The Eulerian rotation has been applied to arrive at (27) where $T$ indicates an element of the following transforming tensors:

$$
\begin{aligned}
& \bar{T}_{x x}=\left[\begin{array}{ccc}
\cos ^{2} \phi_{f} & \sin \phi_{f} \cos \phi_{f} & 0 \\
\sin \phi_{f} \cos \phi_{f} & \sin ^{2} \phi_{f} & 0 \\
0 & 0 & 0
\end{array}\right] \\
& \bar{T}_{y y}=\left[\begin{array}{ccc}
\sin ^{2} \phi_{f} & -\sin \phi_{f} \cos \phi_{f} & 0 \\
-\sin \phi_{f} \cos \phi_{f} & \cos ^{2} \phi_{f} & 0 \\
0 & 0 & 0
\end{array}\right] \\
& \bar{T}_{z z}=\bar{I}-\bar{T}_{x x}-\bar{T}_{y y}=\operatorname{diag}[0,0,1]
\end{aligned}
$$

By substituting the above correlation functions, the dyadic Green's function of the layered media, and the mean fields into (20), the correlations of the scattered field can now be found. Rearranging all the coefficients from the DGF and the mean fields, the scattering coefficients can be written as

$$
\begin{gathered}
\sigma_{\mu \tau \nu \kappa}=\pi k_{0}^{4} \int_{0}^{\pi} d \psi_{f} \int_{0}^{2 \pi} d \phi_{f} p_{1}\left(\psi_{f}, \phi_{f}\right) \\
\cdot \sum_{a, b, c, d}^{-1,1} \sum_{j, k, l, m}^{x, y, z} \Psi_{1 \mu \tau, j k}^{a b} \Psi_{1 \nu \kappa, l m}^{c d^{*}} I_{1 j k l m}^{a b c d}+\pi k_{0}^{4} \\
\cdot \int_{0}^{2 \pi} d \phi_{f} p_{2}\left(\phi_{f}\right) \sum_{p, q, r, s, s, k, l, m}^{\substack{o u, o d \\
e \mu, e d}} \sum_{2 \mu, y, z}^{x, j} \Psi_{2 \mu, j k}^{p q} \Psi_{2 \nu \kappa, l m}^{r s^{*}} I_{2 j k l m}^{p q r s}
\end{gathered}
$$

The scattering contribution of the first term in (29) from region 1 due to the spheroids has been obtained [Nghiem et al., 1993], and the solution consists of 16 terms for downgoing and upgoing incident and scattered waves. Since region 2 is effectively anisotropic, incident and scattered waves can be ordinary or extraordinary and going down or up; these give rise to 216 terms in the second term of (29). All $\Psi_{2}$ coefficients have been derived [Nghiem et al., 1990]. For $I_{2 j k l m}^{\text {prs }}$, the result is

$$
\begin{aligned}
& I_{2 j k l m}^{p q r s}=i \frac{2 \delta_{2, j i m} / m x^{\prime} / 2 y^{\prime} / 2 z^{\prime}}{\pi l_{2 z^{\prime}}^{4}} \\
& +\left[\frac{\exp \left[-i\left(\kappa_{p q}-\kappa_{r s}\right) d_{2}\right]}{\left(\kappa_{p q}-\kappa_{2}\right)^{2}\left(\kappa_{p q}-\kappa_{2}^{*}\right)^{2}\left(\kappa_{p q}-\kappa_{r s}\right)}\right. \\
& +\frac{\exp \left[i\left(\kappa_{r s}-\kappa_{p q}\right) d_{1}\right]}{\left(\kappa_{r s}-\kappa_{2}\right)^{2}\left(\kappa_{r s}-\kappa_{2}^{*}\right)^{2}\left(\kappa_{r s}-\kappa_{p q}\right)} \\
& \left.-P_{2}\left(\kappa_{2}\right)-Q_{2}\left(\kappa_{2}^{*}\right)\right]
\end{aligned}
$$

where $P_{2}\left(\kappa_{2}\right)$ and $Q_{2}\left(\kappa_{2}^{*}\right)$, resulting from complex integrations, are determined by

$$
\begin{aligned}
& P_{2}\left(\kappa_{2}\right) \\
& =\frac{i\left(d_{2}-d_{1}\right) \exp \left[-i\left(\kappa_{2}-\kappa_{r s}\right) d_{1}\right] \exp \left[i\left(\kappa_{2}-\kappa_{p q}\right) d_{2}\right]}{\left(2 i \operatorname{Im} \kappa_{2}\right)^{2}\left(\kappa_{2}-\kappa_{p q}\right)\left(\kappa_{2}-\kappa_{r s}\right)} \\
& +\left[\frac{\exp \left[-i\left(\kappa_{p q}-\kappa_{r s}\right) d_{1}\right]+\exp \left[-i\left(\kappa_{p q}-\kappa_{r s}\right) d_{2}\right]}{\left(2 i \operatorname{Im} \kappa_{2}\right)^{2}\left(\kappa_{2}-\kappa_{p q}\right)\left(\kappa_{2}-\kappa_{r s}\right)}\right. \\
& -\frac{\exp \left[-i\left(\kappa_{2}-\kappa_{r s}\right) d_{1}\right] \exp \left[i\left(\kappa_{2}-\kappa_{p q}\right) d_{2}\right]}{\left(2 i \operatorname{Im} \kappa_{2}\right)^{2}\left(\kappa_{2}-\kappa_{p q}\right)\left(\kappa_{2}-\kappa_{r s}\right)}(30 b) \\
& \quad \cdot\left[\frac{1}{i \operatorname{Im} \kappa_{2}}+\frac{1}{\kappa_{2}-\kappa_{p q}}+\frac{1}{\kappa_{2}-\kappa_{r s}}\right] \\
& Q_{2}\left(\kappa_{2}^{*}\right) \\
& \quad=\frac{i\left(d_{2}-d_{1}\right) \exp \left[i\left(k_{2}^{*}-\kappa_{p q}\right) d_{1}\right] \exp \left[-i\left(\kappa_{2}^{*}-\kappa_{r s}\right) d_{2}\right]}{\left(2 i \operatorname{Im} \kappa_{2}^{*}\right)^{2}\left(\kappa_{2}^{*}-\kappa_{p q}\right)\left(\kappa_{2}^{*}-\kappa_{r s}\right)} \\
& +\frac{\exp \left[i\left(\kappa_{2}^{*}-\kappa_{p q}\right) d_{1}\right] \exp \left[-i\left(\kappa_{2}^{*}-\kappa_{r s}\right) d_{2}\right]}{\left(2 i \operatorname{Im} \kappa_{2}^{*}\right)^{2}\left(\kappa_{2}^{*}-\kappa_{p q}\right)\left(\kappa_{2}^{*}-\kappa_{r s}\right)} \\
& \quad \cdot\left[\frac{1}{i \operatorname{Im} \kappa_{2}^{*}}+\frac{1}{\kappa_{2}^{*}-\kappa_{p q}}+\frac{1}{\kappa_{2}^{*}-\kappa_{r s}}\right]
\end{aligned}
$$

In (30) the pole $\kappa_{2}$ is computed with the following equations:

$$
\begin{aligned}
& \kappa_{2}=i l_{2 z^{\prime}}^{-1} \sqrt{1+k_{\phi x}^{2} l_{2 x^{\prime}}^{2}+k_{\phi y}^{2} l_{2 y^{\prime}}^{2}} \\
& k_{\phi x}=k_{\rho i} \cos \left(\phi_{i}-\phi_{f}\right)-k_{\rho s} \cos \left(\phi_{s}-\phi_{f}\right) \\
& k_{\phi y}=k_{\rho i} \sin \left(\phi_{i}-\phi_{f}\right)-k_{\rho s} \sin \left(\phi_{s}-\phi_{f}\right)
\end{aligned}
$$




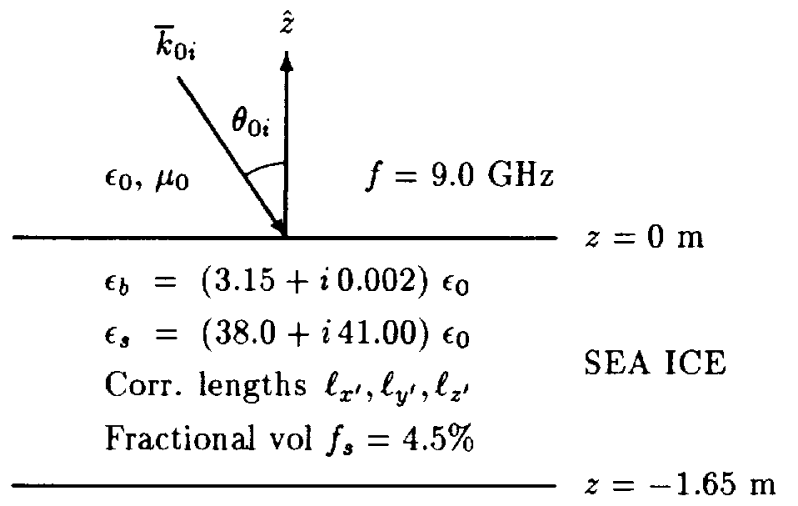

Underlying medium :

$\epsilon=(45.0+i 40.00) \epsilon_{0}$

SEA WATER

Fig. 4. Physical configuration of bare thick first-year sea ice with smooth surface.

where $\phi_{i}$ and $\phi_{s}$ are azimuthal angles of incident and scattered waves, respectively. The remaining integrations over Eulerian angles in (29) can be carried out numerically. When the low-frequency condition is valid, the integrations can be done analytically. It should be noted that (29) expresses the scattering coefficients in the scattered basis which can Be transformed to the incident basis by changing the sign $\sigma_{h \pi / \kappa}$ and $\sigma_{v /{ }^{\prime} \kappa}$ [Nghiem et al., 1990]. Scattering effects of rough boundaries at medium interfaces can also be estimated by incoherently adding the contribution from the roughsurface scattering with consideration of the propagation loss in the calculations of scattering coefficients. Compared with the model for the case of vertically oriented spheroids [Nghiem et al., 1990], the new model can give higher copolarized backscattering coefficients due to higher total cross section of the ellipsoids for the same fractional volume. Another difference is that the new model provides nonzero cross-polarized backscattering in the first-order distorted Born term, whereas the former can only account for the cross-polarized return in the second or higher-order term when the spheroids are vertically oriented.

\section{RESULTS AND DISCUSSION}

\subsection{Data comparisons}

In this subsection, theoretical results are compared with experimental measurements for back- scattering coefficient at $9 \mathrm{GHz}$ as a function of incident angles. Radar backscatter data were collected for thick first-year sea ice near Point Barrow, Alaska [Kim et al., 1984]. For the sea ice with a bare surface, the configuration is illustrated in Figure 4 , representing a sea-ice layer over seawater. The ice layer was $1.65 \mathrm{~m}$ thick and contained brine inclusions. From sea-ice characterization data taken at Point Barrow, Lin [1988] obtained brine permittivity $\varepsilon_{s 1}=(38.0+41.0) \varepsilon_{0}$ (refer to Vant et al. [1978] and Stogryn and Desargant [1985]) and fractional volume $f_{s 1}=4.5 \%$ (from equations by Frankenstein and Gardner [1967]). The background ice permittivity was estimated to be $\varepsilon_{b 1}=(3.15+$ $i 0.002) \varepsilon_{0}$ and the permittivity of seawater $\varepsilon_{2}=(45.0$ + i40.0) $\varepsilon_{0}$. With correlation length $l_{1 x^{\prime}}=0.70 \mathrm{~mm}$, $l_{1 y^{\prime}}=0.25 \mathrm{~mm}$, and $l_{1 z^{\prime}}=1.20 \mathrm{~mm}$, theoretical results match well with the experimental data, as shown in Figure 5, for copolarized backscattering coefficient $\sigma_{h h}$ and $\sigma_{y,}$ at large incident angles, and cross-polarized backscattering coefficient $\sigma_{h v}$ over the range of incident angles.

The disagreement in the copolarized backscattering coefficients at small incident angles is due to the scattering from rough surface. In the configuration of Figure 4, the interfaces have been assumed to be smooth and the rough-surface scattering has been ignored. Effects of the roughness at the lower interface of the thick first-year sea ice are negligible since electromagnetic waves at $9 \mathrm{GHz}$ are attenuated before the seawater is reached. To account for rough-surface effects at the top interface, a Gaussian roughness is now considered with height standard deviation $\sigma_{s}=0.6 \mathrm{~mm}$ and surface correlation

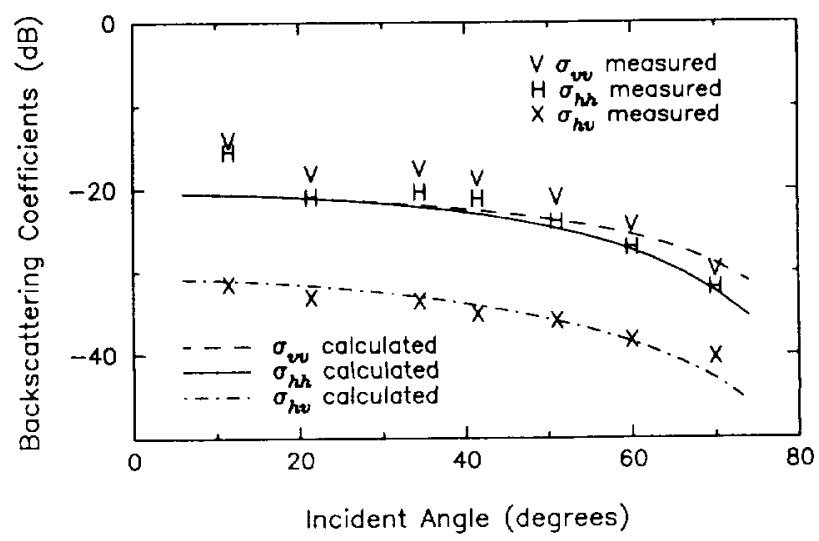

Fig. 5. Backscattering coefficients from bare thick first-year sea ice with smooth surface. 
length $l_{s}=1.5 \mathrm{~cm}$ as depicted in Figure 6, where all other physical parameters are the same as in Figure 4. The rough-surface contribution, calculated with the small perturbation method (SPM) [Tsang et al., 1985], is incoherently added to the volume scattering. The comparison between theoretical and experimental results for the copolarized returns is improved at the low incident angles as seen in Figure 7. For this surface the additional contribution to the cross-polarized return is small and is actually ignored in the SPM model applied in this case. The crosspolarized return therefore remains unchanged.

The configuration for snow-covered sea ice is shown in Figure 8. The snow is $10 \mathrm{~cm}$ thick according to the average of the reported thickness range [Kim et al., 1984]. The fractional volume of ice grains in snow is $34 \%$ [ $\operatorname{Lin}, 1988$ ]. In the snow layer the oblate spheroidal shape is assumed for the ice grains with correlation length $l_{\rho}=l_{z} / 6=0.15 \mathrm{~mm}$. Physical parameters for the sea-ice layer and the seawater are kept unchanged. Both the top and the middle interfaces are rough with standard deviation $\sigma_{s}=0.1 \mathrm{~cm}$ and correlation length $l_{s}=1.5 \mathrm{~cm}$. Theoretical results compare well with measurements from the snow-covered sea ice as seen in Figure 9 for conventional backscattering coefficients. Theoretical cross-polarized returns are, however, lower than the measurements. For a medium with high fractional volume such as snow, a contribution to $\sigma_{h v}$ can come from higher-order scattering which is ignored in the present model.

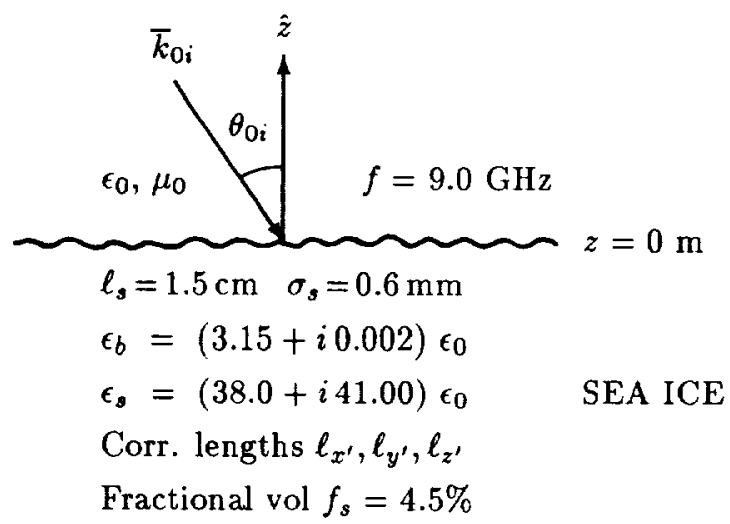

Underlying medium :

$\epsilon=(45.0+i 40.00) \epsilon_{0}$

SEA WATER

Fig. 6. Physical configuration of bare thick first-year sea ice with rough surface.

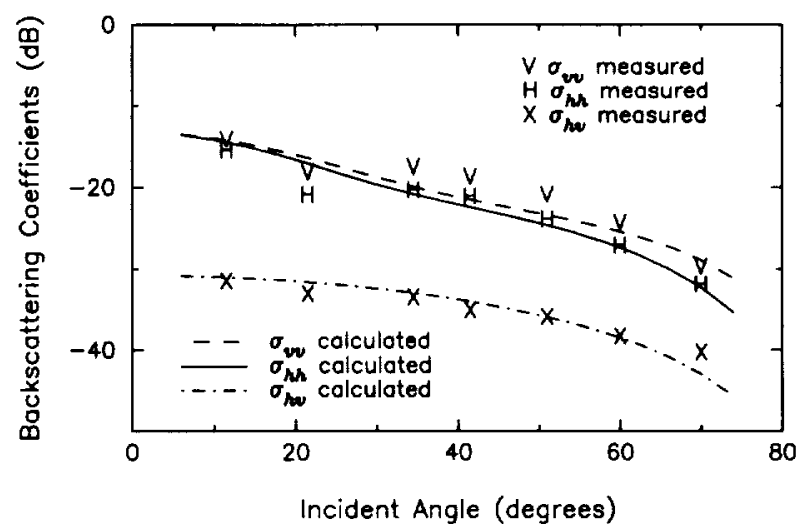

Fig. 7. Backscattering coefficients from bare thick first-year sea ice with rough surface.

Regarding the scattering mechanisms, volume scattering is dominant at large incident angles. Roughsurface effects are strong at small incident angles with more contribution from the snow-ice interface due to the larger permittivity contrast. Oscillations seen in the theoretical curves in Figure 9 are caused

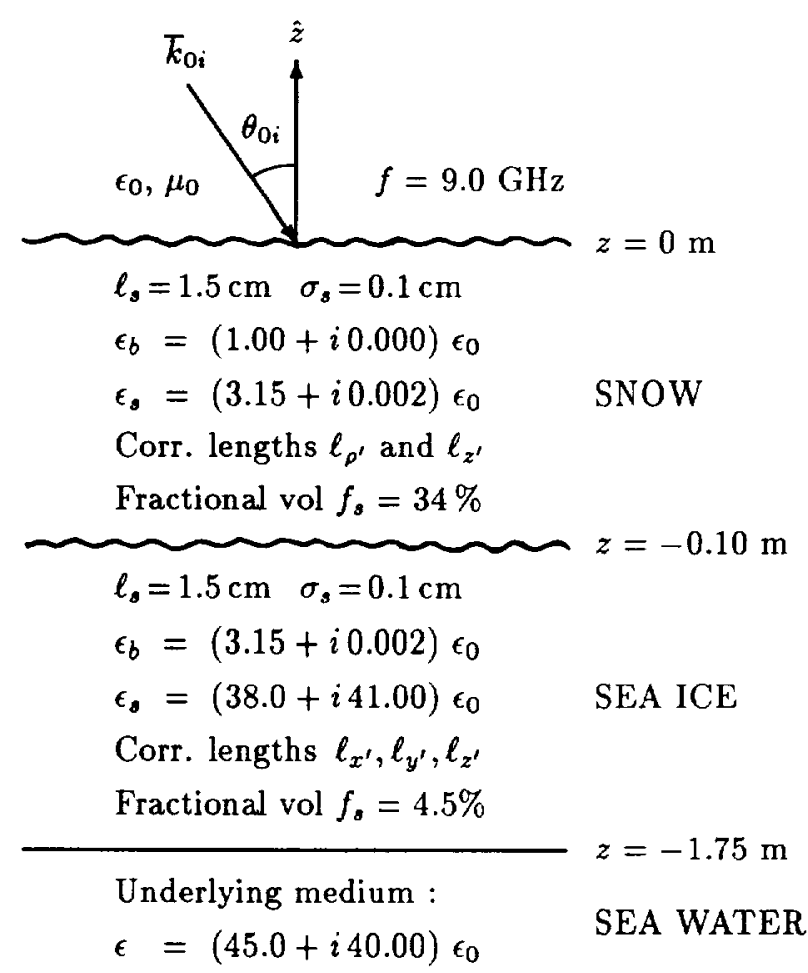

Fig. 8. Physical configuration of thick first-year sea ice with snow cover. 


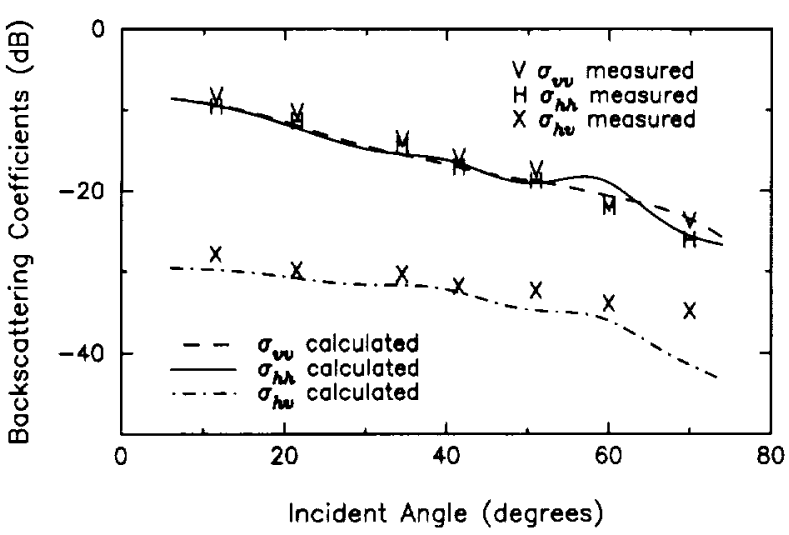

Fig. 9. Backscattering coefficients from thick first-year sea ice with snow cover.

by the boundary effect of the snow cover. Also, the theoretical oscillations in $\sigma_{h h}$ are stronger than those in $\sigma_{h v}$ and those in $\sigma_{v v}$ are weakest. This is because stronger reflections of horizontal waves, due to the medium boundaries, make the wave interference more effective. Comparing the cases with and without snow cover shows that the lowloss dry-snow layer can enhance the backscattering due to the introduction of more scatterers (ice grains) and a better impedance match between the air and the sea-ice layer.

The above results show that the scattering from the inhomogeneities is dominant at large incident angles, while the contribution from the rough surface is important at small incident angles for copolarized returns. The cross-polarized return, which occurs as a result of the nonspherical scatterers, comes from the first-order term under the distorted Born approximation in this model. In the next few subsections, effects of correlation lengths and polarization signatures of sea ice are considered.

\subsection{Study of correlation lengths}

The geometry of brine inclusions in sea ice is not available from ice characterization data for the Point-Barrow sea ice under consideration. To study effects of the scatterer size and shape, backscattering coefficients are calculated with variable correlation lengths. To isolate effects of correlation lengths, bare sea ice with smooth surface configuration in Figure 4 is considered to avoid the contribution from the snow layer and the rough interfaces. The correlation lengths used in the data matching serve as a reference. The scatterer is expanded, contracted, thickened, thinned, elongated, and shortened by varying the correlation lengths as shown in Table 1, while the fractional volume is kept constant in all cases.

When all correlation lengths are varied by the same factor, the scatterer shape remains unchanged since the correlation length ratios stay the same. For the same fractional volume, the effective number of scatterers decreases as the scatterer is expanded and increases when the scatterer is contracted. Conventional backscattering coefficients as well as correlation coefficient $\rho=\sigma_{h h r v} /\left(\sigma_{h h} \sigma_{v v}\right)^{1 / 2}$ [Nghiem et al., 1990] between horizontal and vertical returns are calculated for the volume expansion and contraction with the same factor of $1.5^{3}$. The results are plotted in Figure 10. For $\sigma_{h h}$ and $\sigma_{v v}$, the scattering coefficients are reduced much more when the correlation volume of the scatterer is contracted as compared to the increases in the case of volume expansion. This shows a nonlinear relationship between volume scattering and the scatterer size. In this case an underestimate in the scatterer volume, compared to the overestimate with the same factor, will lead to more variations in copolarized backscattering coefficients. An interesting observation is the insensitivity of cross-

TABLE 1. Correlation Lengths for Various Scatterer Shapes

\begin{tabular}{lccccccc}
\hline SHAPE & $l_{x}^{\prime}$ & $l_{y}^{\prime}$ & $l_{z}^{\prime}$ & $l_{x}^{\prime} / l_{y}^{\prime}$ & $l_{z}^{\prime} / l_{x}^{\prime}$ & $l_{z}^{\prime} / l_{y}^{\prime}$ & $l_{x}^{\prime} l_{y}^{\prime} l_{z}^{\prime}$ \\
\hline Reference & 0.700 & 0.250 & 1.200 & 2.800 & 1.714 & 4.800 & 0.210 \\
Expanded & 1.050 & 0.375 & 1.800 & 2.800 & 1.714 & 4.800 & 0.709 \\
Contracted & 0.467 & 0.167 & 0.800 & 2.800 & 1.714 & 4.800 & 0.062 \\
Thickened & 0.467 & 0.375 & 1.200 & 1.245 & 2.570 & 3.200 & 0.210 \\
Thinned & 1.050 & 0.167 & 1.200 & 6.287 & 1.143 & 7.186 & 0.210 \\
Elongated & 0.572 & 0.204 & 1.800 & 2.800 & 3.147 & 8.824 & 0.210 \\
Shortened & 0.857 & 0.306 & 0.800 & 2.800 & 0.933 & 2.614 & 0.210 \\
\hline
\end{tabular}



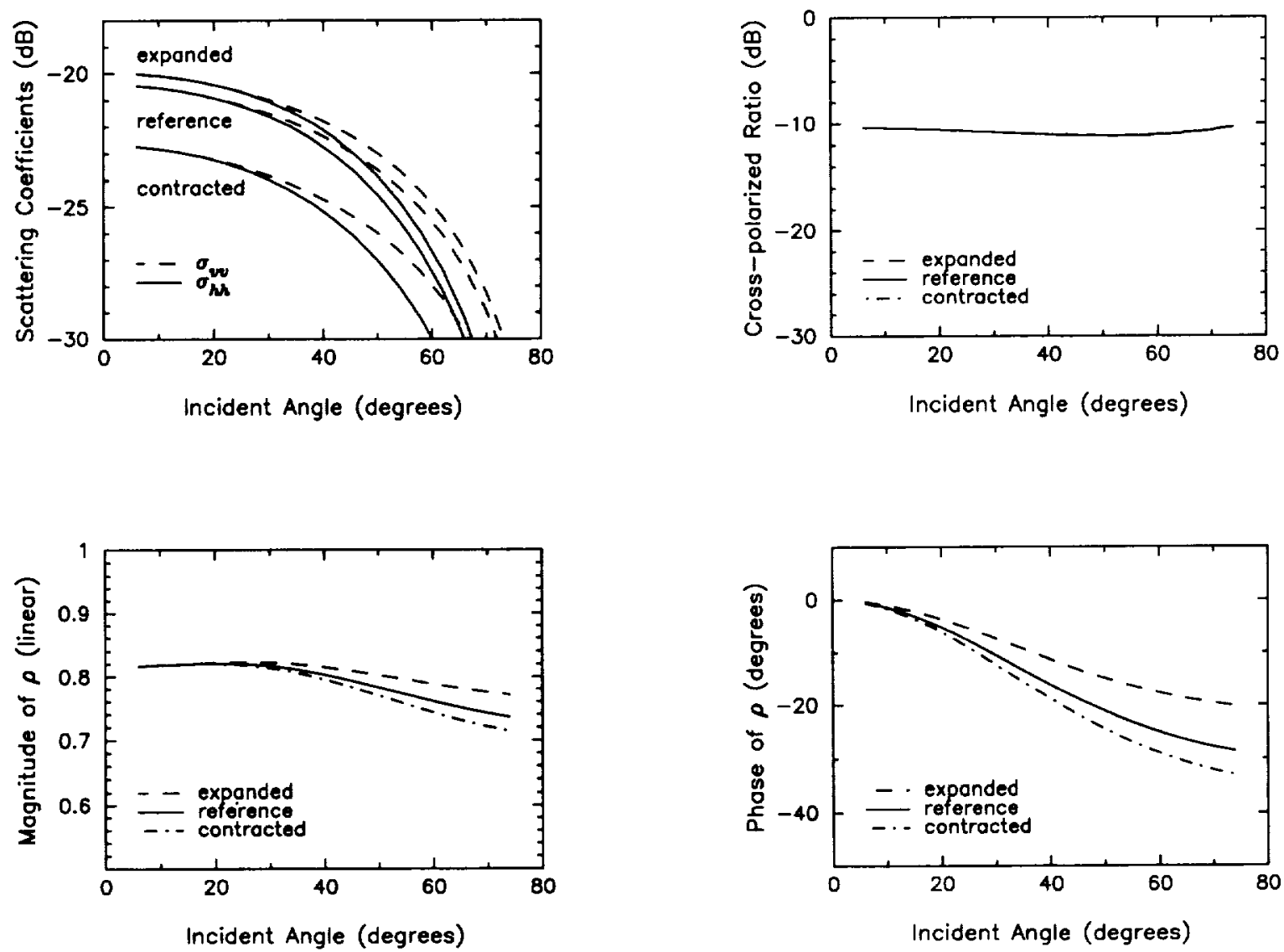

Fig. 10. Effects of expanded and contracted scatterer volumes.

polarized ratio $e=\sigma_{h v} / \sigma_{h h}$ as shown in Figure 10 . This is because the depolarization is caused by the nonspherical shape of the scatterer, which is kept unchanged. For correlation coefficient $\rho$, the results show that horizontal and vertical waves become more correlated as the correlation volume increases. For larger volume of the scatterer, the sea ice medium is more lossy and the wave path is effectively reduced. Consequently, the anisotropic effect causing the decorrelation between horizontal and vertical waves is weakened.

When correlation length ratios are varied while the volume of an individual scatterer and the total fractional volume are kept constant, the scatterer shape is deformed and the number of scatterers remains unchanged. For the same vertical correlation length $l_{z^{\prime}}$, horizontal correlation lengths are now modified by a factor of 1.5 to thicken and to thin the scatterer as indicated in Table 1. In Figure
11, copolarized returns are rather insensitive, since the horizontal cross section of the scatterer is not varied $\left(l_{x^{\prime}} l_{z^{\prime}}=0.175 \mathrm{~mm}^{2}\right)$. It is seen in Figure 11 that the cross-polarized ratio is strongly dependent on the scatterer shape. The depolarization effect is more prominent, as the scatterer is further deformed from the spheroidal shape. For the magnitude of $\rho$, the variation is larger at small incident angles where the scatterer shape is more different as compared to the cases at large incident angles. The phase of $\rho$ started from $0^{\circ}$ at normal incidence due to the azimuthal symmetry [Nghiem et al., 1992] and increases with incident angles. Also, the absolute value of the phase is larger for the thicker scatterer since the anisotropy is stronger.

While the correlation length ratio $l_{x^{\prime}} / l_{y^{\prime}}$, the number of scatterers, and the fractional volume are not changed, the scatterer is elongated and shortened by varying vertical correlation length $l_{z^{\prime}}$ by a factor 

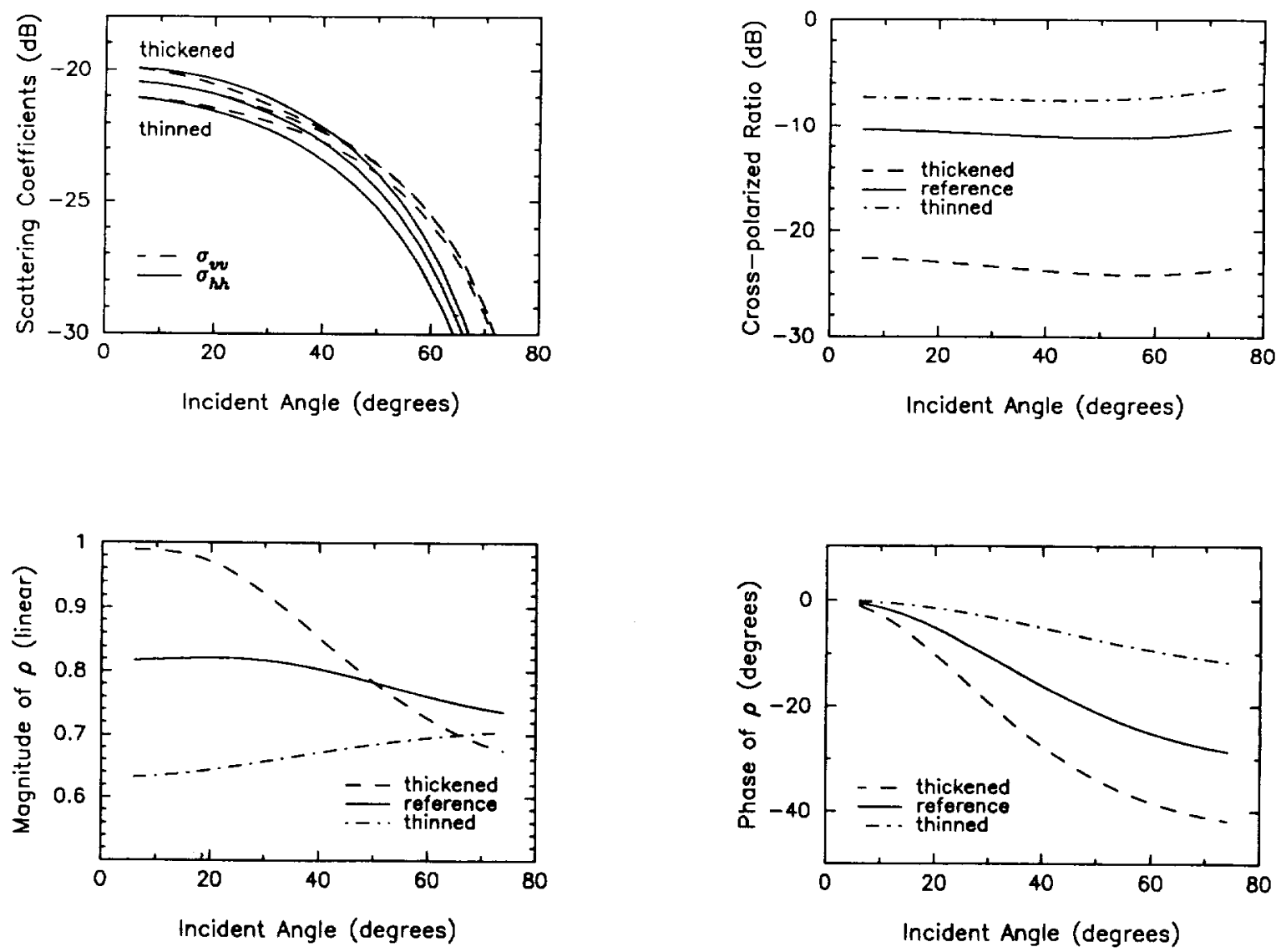

Fig. 11. Effects of thickened and thinned scatterer shapes.

of 1.5 . Figure 12 presents results for these cases compared with the reference case. There are large changes in copolarized returns due to the corresponding differences in the horizontal cross section of the scatterer. The variation in the cross-polarized ratio is small, since horizontal correlation length ratio $l_{x^{\prime}} / l_{y^{\prime}}$ is not varied. The magnitude of $\rho$ differs more at large incident angles, where the shape difference is more significant. The phase of $\rho$ is closer to zero as the scatterer becomes shorter and the sea-ice medium approaches the isotropic condition. As shown in this subsection, scatterer volume and horizontal cross section affect the level of copolarized returns. The depolarization effect characterized by cross-polarized ratio $e$ is controlled by the scatterer shape. The ellipsoidal shape of the scatterer with a preferential alignment in the vertical direction is also responsible for the anisotropy of the medium and, consequently, the behavior of complex correlation coefficient $\rho$ between horizontal and vertical returns.

\subsection{Polarimetric simulation}

To investigate how polarization signatures relate to the corresponding covariance matrices and convey information regarding different layered configurations and scattering mechanisms, polarimetric data are simulated for sea ice with a bare surface and with a snow cover at different incident angles. Definitions and characteristics of covariance matrix and polarization signature can be found in references [Nghiem et al., 1990; van Zyl and Zebker, 1990]. The configurations under consideration are in Figure 4 for bare sea ice with a smooth surface, in Figure 6 for bare ice with a rough surface, and in 

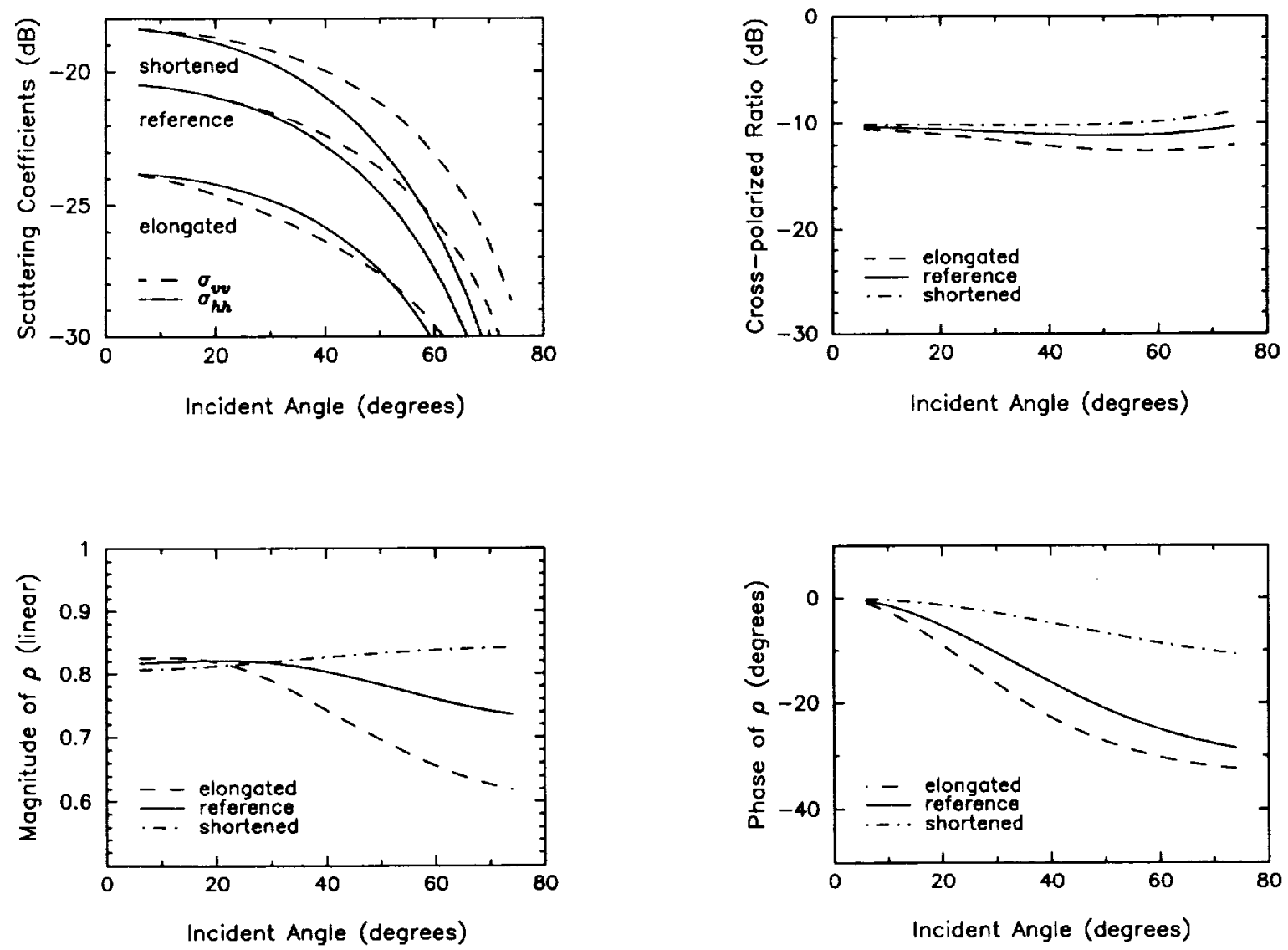

Fig. 12. Effects of elongated and shortened scatterer shapes.

Figure 8 for snow-covered sea ice. As a result of the azimuthal symmetry of the scattering configuration, the covariance matrix has the form of [Nghiem et al., 1992]

$$
\bar{C}=\sigma_{h h}\left[\begin{array}{ccc}
1 & 0 & \rho \sqrt{\gamma} \\
0 & e & 0 \\
\rho^{*} \sqrt{\gamma} & 0 & \gamma
\end{array}\right]
$$

where the copolarized ratio is $\gamma=\sigma_{v v} / \sigma_{h h}$. Note that covariance matrix $\overline{\bar{C}}$ (without subscript) should be distinguished from correlation tensor $\bar{C}_{\xi n}$ which is tagged with subscript $\xi$, corresponding to correlation function $\Phi_{\xi}$ and subscript $n$ for region $n=1$, 2. Elements of the covariance matrix are used to calculate the Mueller matrix and to obtain the polarization signature $\sigma\left(\alpha_{r}, \beta_{r}, \alpha_{i}, \beta_{i}\right)[\mathrm{Nghiem}$ et al., 1990; van Zyl and Zebker, 1990] for polarization angles $\alpha_{r}$ and $\alpha_{i}$ and ellipticity angles $\beta_{r}$ and $\beta_{i}$ of the received $(r)$ and the incident $(i)$ waves, respectively. For a copolarized signature, $\alpha_{r}=\alpha_{i}=\alpha$ and $\beta_{r}=\beta_{i}=\beta$.

Normalized copolarized signatures denoted by $\sigma_{n}$ [Nghiem et al., 1990] for bare sea ice with smooth and rough surfaces at incident angles of $20^{\circ}$ and $45^{\circ}$ are presented in Figure 13. At $\theta_{0 i}=20^{\circ}$, signatures for smooth sea ice due to volume scattering and for rough sea ice due to surface scattering are similar because both covariance matrices have $y$ close to unity, small $e$, and $\rho$ with small phase as seen in Figure 14. However, $|\rho|$ shown in Figure 14 is distinctively smaller for the volume-scattering mechanism with smooth surface. At $\theta_{0 i}=45^{\circ}$ for both smooth- and rough-surface cases, volume scattering is dominant and correlation coefficients with larger phase give rise to signatures in Figures $13 c$ and $13 d$ with more distortion compared to those at 

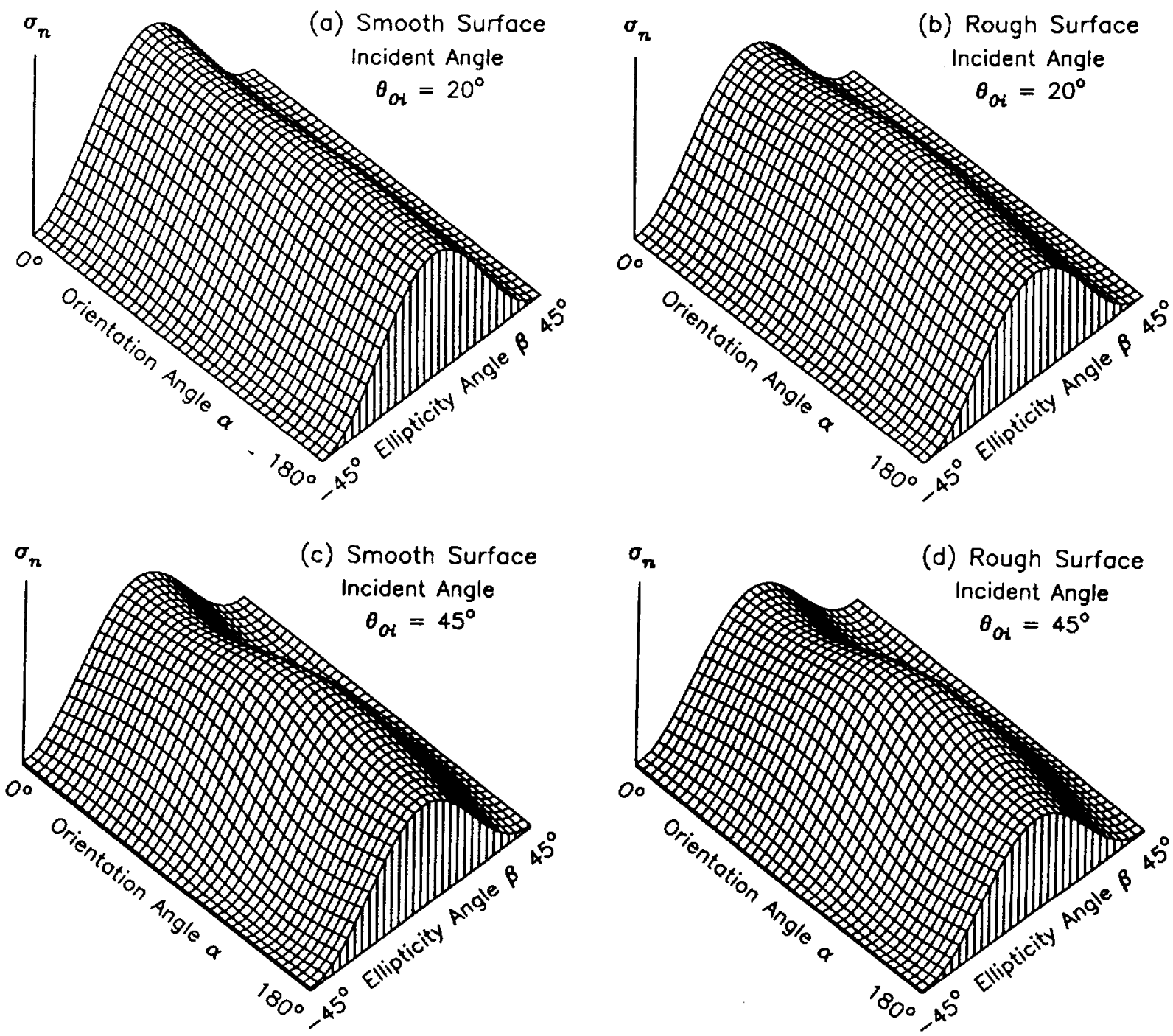

Fig. 13. Polarization signatures of bare thick first-year sea ice with $(a)$ a smooth surface with an incident angle $\left(\theta_{0 i}\right)$ of $20^{\circ},(b)$ a rough surface at incident angle of $20^{\circ},(c)$ a smooth surface with an incident angle of $45^{\circ}$, and $(d)$ a rough surface with an incident angle of $45^{\circ}$.

$20^{\circ}$. For snow-covered sea ice, Figure 14 reveals that $|\rho|$ is closer to unity, and the phase is smaller at incident angles of $20^{\circ}$ and $45^{\circ}$. Thus the corresponding signatures for the snow-covered sea ice in Figure 15 have much less distortion due to the rough surfaces and the isotropic snow layer which mask the anisotropic information from the lower sea-ice layer at the $X$ band frequency. Boundary effects due to snow cover can also be seen with the oscillations in the phase of $\rho$ in Figure 14.

\section{SUMMARY}

In this paper a model has been developed to account for ellipsoidal shapes of scatterers in anisotropic layered media. Ellipsoidal scatterers with a preferential alignment in the vertical direction have been observed in horizontal and vertical sections of sea ice. Effective permittivities are derived with the extended strong fluctuation theory, and fully polarimetric backscattering coefficients are calculated 

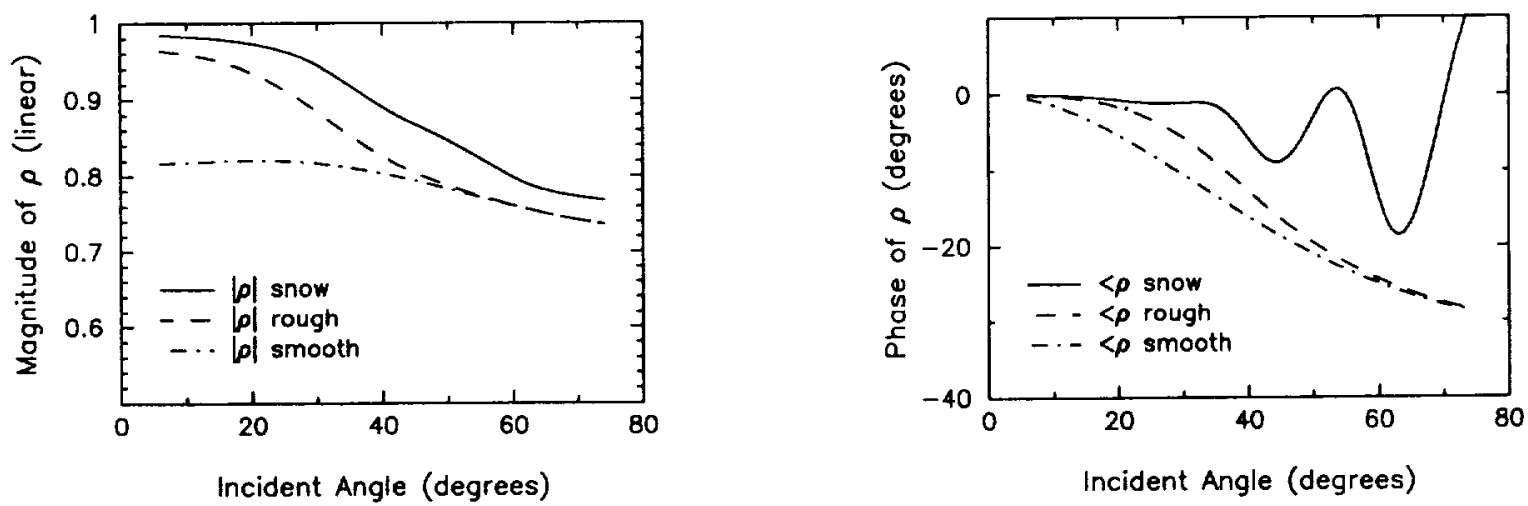

Fig. 14. Correlation coefficient $\rho$ of thick first-year sea ice with smooth surface, rough surface, and snow cover.

under the distorted Born approximation. In this model the cross-polarized return is obtained from the first-order approximation due to nonspherical shapes of the scatterers. Effects of rough surfaces are estimated with the incoherent addition method. Theoretical results compare well with measured data at $9 \mathrm{GHz}$ for thick first-year sea ice with a bare surface and a snow cover. Changes in scattering coefficients due to the scatterer geometry are investigated by varying correlation lengths which modify the shape or the volume of the scatterer. This sensitivity study also provides an estimation of variations in polarimetric scattering coefficients due to uncertainties in the scatterer sizes and shapes. Polarization signatures are simulated for the thick first-year sea ice with and without snow cover. These signatures convey information on the medium anisotropy, which are influenced by rough-surface or snow cover effects. Volume- and surface-scattering mechanisms are discussed in terms of conventional backscattering coefficients as well as polarization signatures and correlation coefficient $\rho$ between vertical and horizontal returns. Further medium complexity such as multispecies, size and shape distributions, and higherorder volume or surface scattering have not been incorporated in this model and can be considered for future model developments with comparisons to well-calibrated measurements of polarimetric covariance matrices.
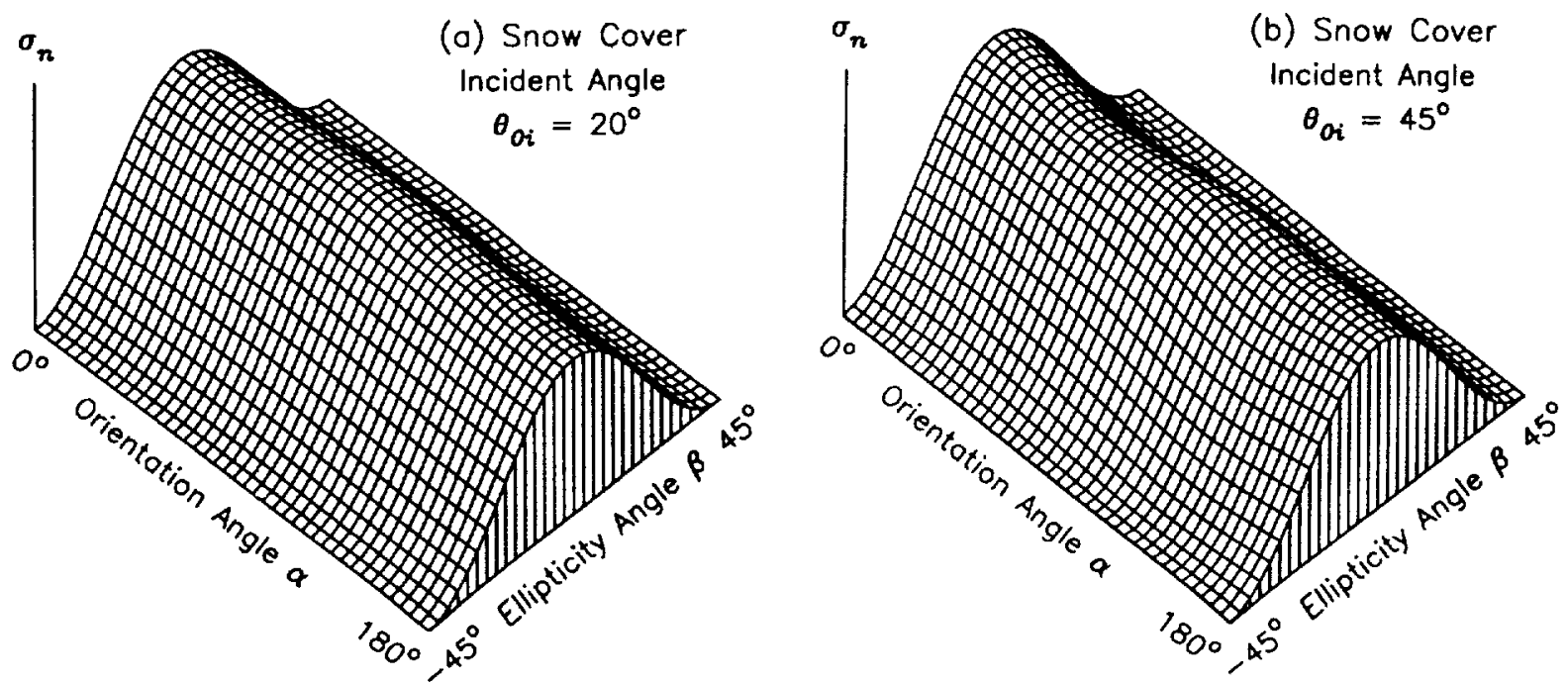

Fig. 15. Polarization signatures of thick first-year sea ice with snow cover at incident angles of $20^{\circ}$ and $45^{\circ}$. 
Acknowlegments. The research described in this paper was carried out by the Jet Propulsion Laboratory, California Institute of Technology, and was sponsored by the Office of Naval Research and the National Aeronautics and Space Administration; and by the Massachusetts Institute of Technology, under contract N00014-89-J-1107 with the Office of Naval Research.

\section{REFERENCES}

Borgeaud, M., J. A. Kong, and F. C. Lin, Microwave remote sensing of snow-covered sea ice, Proc. IGARSS'86 Remote Sens. Symp., 133-138, 1986.

Borgeaud, M., R. T. Shin, and J. A. Kong, Theoretical models for polarimetric radar clutter, J. Electromagn. Waves Appl., I(1), 73-89, 1987.

Borgeaud, M., S. V. Nghiem, R. T. Shin, and J. A. Kong, Theoretical models for polarimetric microwave remote sensing of earth terrain, J. Electromagn. Waves Appl, 3(1), 61-81, 1989.

de Wolf, D. A., Electromagnetic reflection from an extended turbulent medium: Cumulative forward-scatter single backscatter approximation, IEEE Trans. Antennas Propag., 19(2), 254-262, 1971.

Frankenstein, G., and R. Garner, Equation for determining the brine volume of sea ice from $-0.5^{\circ}$ to $-22.9^{\circ} \mathrm{C}, J$. Glaciol., 6(48), 943-944, 1967.

Gow, A. J., S. A. Arcone, and S. G. McGrew, Microwave and structure properties of saline ice, CRREL Rep. 87-20, U.S. Army Corps of Engineers, Cold Regions Research and Engineering Laboratory, Hanover, N. H., 1987.

Kim, Y. S., R. K. Moore, and R. G. Onstott, Theoretical and experimental study of radar backscatter from sea ice, $R S L$ Tech. Rep. 331-37, Remote Sens. Lab., Cent. for Res., Inc., Univ. of Kansas, Lawrence, 1984.

Lang, R. H., Electromagnetic backscattering from a sparse distribution of lossy dielectric scatterers, Radio Sci., 16(1), 15-30, 1981.

Lee, J. K., and J. A. Kong. Active microwave remote sensing of an anisotropic random medium layer, IEEE Trans. Geosci. Remote Sens., 23(6), 910-923, 1985.

Lin, F. C., J. A. Kong, and R. T. Shin, Theoretical models for microwave remote sensing of snow-covered sea ice, Proceedings of the International Geoscience and Remote Sensing Symposium, Institute of Electrical and Electronics Engineers, New York, 1987.

Lin, F. C., Theoretical models for microwave remote sensing of snow-covered sea ice, Ph.D. dissertation, Dep. of Electr. Eng. and Comput. Sci., Mass. Inst. of Technol., Cambridge, 1988.

Nghiem, S. V., M. Borgeaud, J. A. Kong, and R. T. Shin, Polarimetric remote sensing of geophysical media with layer random medium model, Progress in Electromagnetics $R e$ search, vol. 3, Polarimetric Remote Sensing, chap. 1, edited by J. A. Kong, pp. 1-73, Elsevier, New York, 1990.

Nghiem, S. V., S. H. Yueh, R. Kwok, and F. K. Li, Symmetry properties in polarimetric remote sensing, Radio $S c i ., 27(5)$, 693-711, 1992.

Nghiem, S. V., T. Le Toan, J. A. Kong, H. C. Han, and M. Borgeaud, Layer model with random spheroidal scatterers for remote sensing of vegetation canopy, J. Electromagn. Waves Appl., 7(1), 49-76, 1993.

Rosenbaum, S., and L. W. Bowles, Clutter return from vegetated areas, IEEE Trans. Antennas Propag., 22(2), 227-236, 1974.

Stogryn, A., and G. J. Desargant, The dielectric properties of brine in sea ice at microwave frequencies, IEEE Trans. Antennas Propag., AP-33(5), 523-532, 1985.

Stogryn, A., An analysis of the tensor dielectric constant of sea ice at microwave frequencies, IEEE Trans. Geosci. Remote Sens., $G E-25(2), 147-158,1987$.

Tsang, L., and J. A. Kong, Scattering of electromagnetic waves from random media with strong permittivity fluctuations, Radio Sci., 16(3), 303-320, 1981 a:

Tsang, L., and J. A. Kong, Application of strong fluctuation random medium theory to scattering from vegetation-like half space, IEEE Trans. Geosci. Remote Sens., 19(1), 62-69, $1981 b$.

Tsang, L., J. A. Kong, and R. W. Newton, Application of strong fluctuation random medium theory to scattering of electromagnetic waves from a half-space of dielectric mixture, IEEE Trans. Antennas Propag., 30(2), 292-302, 1982.

Tsang, L., J. A. Kong, and R. T. Shin, Theory of Microwave Remote Sensing, John Wiley, New York, 1985.

van $\mathrm{Zyl}$, J. J., and H. A. Zebker, Imaging radar polarimetry, Progress in Electromagnetics Research, vol. 3, Polarimetric Remote Sensing, chap. 5, edited by J. A. Kong, pp. 277-326, Elsevier, New York, 1990.

Vant, M. R., R. O. Ramseier, V. Makios, The complex dielectric constant of sea ice at frequencies in the range $0.1-40 \mathrm{GHz}, J$. Applied Phys., 49(3), 1264-1280, 1978.

Weeks, W. F., and S. F. Ackley, The Growth, Structure, and Properties of Sea Ice, Monogr. Ser., vol. 82-1, U.S. Army Corps of Engineers, Cold Regions Research and Engineering Laboratory, Hanover, N. H., 1982.

Yueh, H. A., R. T. Shin, and J. A. Kong, Scattering from randomly oriented scatterers with strong permittivity fluctuations, J. Electromagn. Waves Appl., 4(10), 983-1004, 1990.

Zuniga, M. A., T. M. Habashy, and J. A. Kong, Active remote sensing of layered random media, IEEE Trans. Geosci. Electron., 17(4), 296-302, 1979.

Zuniga, M. A., J. A. Kong, and L. Tsang, Depolarization effects in the active remote sensing of random media, J. Applied Phys., 51(5), 2315-2325, 1980.

J. A. Kong and R. T. Shin, Department of Electrical Engineering and Computer Science, Massachusetts Institute of Technology, Cambridge, MA 02139.

R. Kwok and S. V. Nghiem, Jet Propulsion Laboratory, California Institute of Technology, Mail Stop 300-235, 4800 Oak Grove Drive, Pasadena, CA 91109. 
